\title{
The genus Codonopsis (Campanulaceae): a review of phytochemistry, bioactivity and quality control
}

\author{
Jing-Yu He $\cdot \mathrm{Na} \mathrm{Ma} \cdot$ Shu Zhu $\cdot$ Katsuko Komatsu • \\ Zhi-Yuan Li • Wei-Ming Fu
}

Received: 7 May 2014/Accepted: 18 July 2014/Published online: 7 August 2014

(C) The Japanese Society of Pharmacognosy and Springer Japan 2014

\begin{abstract}
Codonopsis, in the family Campanulaceae, is a genus containing 42 species of dicotyledonous herbaceous perennial plants, predominantly found in Central, East and South Asia. Several Codonopsis species are widely used in traditional medicine and are considered to have multiple medicinal properties. Among the Codonopsis species, Codonopsis pilosula (Franch.) Nannf. and C. lanceolata (Sieb. et Zucc.) Benth. \& Hook. f. ex Trautv. are more popular than others according to the findings, especially phytochemical and bioactive studies. Phytochemical research shows that Codonopsis species contain mainly polyacetylenes, phenylpropanoids, alkaloids, triterpenoids and polysaccharides, which contribute to multiple bioactivities. However, the mechanisms of their bioactivities need to be further elucidated. The less popular Codonopsis species remain to be studied and exploited. In addition, although a series of methods for the quality evaluation of Codonopsis species have been developed, a feasible and reliable approach to the efficacious and safe use of various Codonopsis species is still needed, with considering botanical origin, chemical constituents and bioactive effects. This review aims to provide up-to-date and comprehensive information on the phytochemistry, bioactivity and quality control of medicinal plants in the genus Codonopsis and to highlight current gaps in knowledge,
\end{abstract}

J.-Y. He · N. Ma · Z.-Y. Li · W.-M. Fu ( $ه)$

Guangzhou Institute of Advanced Technology, Chinese

Academy of Sciences, 1121 Haibin Rd., Nansha Dist.,

511-458 Guangzhou, People's Republic of China

e-mail:wm.fu@giat.ac.cn

S. Zhu · K. Komatsu

Division of Pharmacognosy, Department of Medicinal

Resources, Institute of Natural Medicine, University of Toyama,

2630 Sugitani, Toyama 930-0194, Japan which is useful for the wider development of the Codonopsis genus.

Keywords Codonopsis - Phytochemistry - Bioactivity · Quality control

\section{Introduction}

Codonopsis, which is a dicotyledonous genus of herbaceous perennial plants in the family Campanulaceae, has 42 species predominantly distributed in Central, East and South Asia; 40 Codonopsis species can be found in China [1]. However, Codonopsis pilosula (Franch.) Nannf., C. pilosula Nannf. var. modesta (Nannf.) L. D. Shen, $C$. tangshen Oliv. and C. lanceolata (Sieb. et Zucc.) Benth. \& Hook. f. ex Trautv. are the only species widely used, of which the fresh or dried roots are collectively regarded as famous herbal medicines and have been used in folk medicine for hundreds of years. Codonopsis Radix is prescribed as the dried roots of $C$. pilosula, C. pilosula var. modesta and $C$. tangshen in the Chinese Pharmacopoeia (2010 edition) [2]. It is called "Dangshen" in Chinese and "Tojin" in Japanese, and has been used in traditional Chinese medicine for replenishing $q i$ (vital energy) deficiency, strengthening the immune system, improving poor gastrointestinal function, gastric ulcer and appetite, decreasing blood pressure, etc., and is sometimes used as a substitute for Ginseng (Panax ginseng C. A. Mey.) [3, 4]. The roots of other Codonopsis species, including C. tubulosa, C. subglobosa, C. clematidea and C. lanceolata, are reported to be used as substitutes for Codonopsis Radix in some regions [3]. C. lanceolata, commonly called bonnet bellflower, is a herb with high value in traditional Chinese medicine and its root is also becoming popular as a 
special vegetable in some Asian countries [5]. C. lanceolata has been used for the treatment of bronchitis, asthma, cough, tuberculosis, dyspepsia and psychoneurosis [6-8]. Phytochemical researches have revealed that the roots of Codonopsis species contained alkaloids, phenylpropanoids, triterpenoids, polyacetylenes, flavones, organic acid, polysaccharides, etc. [9-54]. Among them, polyacetylenes, phenylpropanoids, alkaloids, triterpenoids and polysaccharides are considered to be the major constituents and responsible for most of the activities found in the plants of this genus. The chemical profile varied greatly between species and sample collections may cause these Codonopsis species to possess diverse bioactivities. Some compounds belonging to these five chemotypes have been evaluated for potential biological activity and pharmacological mechanisms. However, the pharmacological mechanisms of these Codonopsis species related to biological activity and clinical application remain largely unexplained. Additionally, the toxicity of Codonopsis has not been reported in the scientific literature.

As many investigations indicated that a variety of chemical constituents contributed to the effects of Codonopsis species, the quantitation of bioactive components becomes urgent for ensuring the efficacy of Codonopsis species. In the Chinese Pharmacopoeia (2010 edition), only lobetyolin is used as the chemical marker for identification of Codonopsis Radix, which seems useless for many Codonopsis species involved [2]. Hence, a number of studies have attempted to develop accurate, sensitive and selective analytical methods for qualitative and quantitative evaluation of Codonopsis materials.

To provide information benefiting traditional uses and scientific studies, this review summarizes and evaluates the available phytochemical and bioactive properties of Codonopsis genus reported by the literature. In addition, for the efficacious and safe uses of Codonopsis, the progress of research on quality evaluation of various Codonopsis species is also presented.

\section{Chemical constituents of genus Codonopsis}

Phytochemical studies have been performed on Codonopsis species plants over the last 30 years all around the world. Only some of the different Codonopsis species plants have been explored for obtaining information on chemo-taxonomical identification, isolation and identification of various important chemicals from this genus and comparison of the chemicals in different plants or species.

C. pilosula, C. tangshen, C. lanceolata and C. clematidea have been widely investigated in their phytochemistry; more than 100 compounds have thereby been isolated and identified. On the other hand, few compounds in $C$. cordifolioidea, C. nervosa, C. thalictrifolia, C. xundianensis and C. tubulosa are reported because they are found only in selected regions. The components in other Codonopsis species have not yet been reported since these Codonopsis species may be difficult to collect and/or be scarce. To date, polyacetylenes, phenylpropanoids, alkaloids, triterpenoids, etc. have been isolated and characterized from the different parts of these Codonopsis species plants. The names of these constituents, the plant and the parts from which they are derived are summarized in Table 1. The structures of the compounds are shown in Figs. 1, 2, 3, 4, 5, 6 and 7.

\section{Alkaloids}

The pyrrolidine alkaloids codonopsine (1), codonopsinine (2), codonopsinol (3) and radicamine A (4) were isolated from the aerial parts of $C$. clematidea $[9,10]$. Two pyrrolidine alkaloids, codonopyrrolidiums A (5) and B (6), were isolated from the roots of $C$. tangshen [13], and were also found in the roots of $C$. pilosula and C. pilosula var. modesta [12, 14]. In addition, the pyrrolidine alkaloids codonopsinols A (7), B (8), C (9) and the glycoside, codonopiloside A (10) were obtained from the roots of $C$. pilosula [11]. Codotubulosine B (11) was found in the roots of C. tubulosa [15].

Other alkaloids, $n$-9-formyl harman (12), norharman (13), 1-carbomethyl carboline (14), 1,2,3,4-tetrahydro- $\beta$ carboline-3-carboxylic acid (15) and tryptophan (16), were isolated from the roots of $C$. lanceolata [16, 17, 19]. Tryptophan (16), perlolyrine (17) and nicotinic acid (18) were obtained from the roots of $C$. pilosula $[18,20,21]$. The common compounds uracil (19) and adenosine (20) were found in the roots of $C$. pilosula and the roots of $C$. pilosula and $C$. tangshen, respectively [13, 18, 22].

\section{Phenylpropanoids}

The phenylpropanoids tangshenosides I (21), II (22), III (23) and IV (24) were first isolated from C. tangshen [23, 25]. Tangshenoside $\mathrm{V}$ (25), tangshenoside VI (26) and codonosides A (27) and B (28), considered to be the characteristic components, were isolated from $C$. tangshen $[13,26]$. Tangshenoside VI (26) was also isolated from the aerial parts of $C$. nervosa [27]. Recently, tangshenoside VIII (29) has been obtained from the roots of $C$. lanceolata [24]. In addition, 12 phenylpropanoids, cordifoliketones A (30) and B (31), sinapinaldehyde (32), coniferaldehyde (33), coniferoside (34), isoconiferin (35), nervolans B (36) and $\mathrm{C}$ (37), dillapiole (38), 1-allyl-2,6-dimethoxy-3,4methylenedioxybenzene (39), 4-allyl-2-(3-methylbut-2-en- 


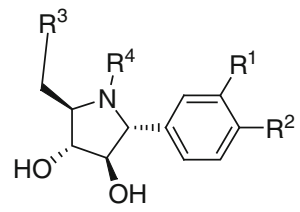

Codonopsine (1): $\mathrm{R}^{1}=\mathrm{OCH}_{3}, \mathrm{R}^{2}=\mathrm{OCH}_{3}, \mathrm{R}^{3}=\mathrm{H}, \mathrm{R}^{4}=\mathrm{CH}_{3}$ Codonopsinine (2): $\mathrm{R}^{1}=\mathrm{H}, \mathrm{R}^{2}=\mathrm{OCH}_{3}, \mathrm{R}^{3}=\mathrm{H}, \mathrm{R}^{4}=\mathrm{CH}_{3}$ Codonopsinol (3): $\mathrm{R}^{1}=\mathrm{OCH}_{3}, \mathrm{R}^{2}=\mathrm{OCH}_{3}, \mathrm{R}^{3}=\mathrm{OH}, \mathrm{R}^{4}=\mathrm{CH}_{3}$ Radicamine A (4): $\mathrm{R}^{1}=\mathrm{OCH}_{3}, \mathrm{R}^{2}=\mathrm{R}^{3}=\mathrm{OH}, \mathrm{R}^{4}=\mathrm{H}$ Codonopsinol A (7): $\mathrm{R}^{1}=\mathrm{OCH}_{3}, \mathrm{R}^{2}=\mathrm{R}^{3}=\mathrm{OH}, \mathrm{R}^{4}=\mathrm{CH}_{3}$ Codonopsinol B (8): $\mathrm{R}^{1}=\mathrm{OCH}_{3}, \mathrm{R}^{2}=\mathrm{H}, \mathrm{R}^{3}=\mathrm{OH}, \mathrm{R}^{4}=\mathrm{CH}_{3}$ Codonopsinol C (9): $\mathrm{R}^{1}=\mathrm{OH}, \mathrm{R}^{2}=\mathrm{H}, \mathrm{R}^{3}=\mathrm{OH}, \mathrm{R}^{4}=\mathrm{CH}_{3}$ Codonopiloside A (10): $\mathrm{R}^{1}=\mathrm{OCH}_{3}, \mathrm{R}^{2}=\mathrm{H}, \mathrm{R}^{3}=\mathrm{O}-\mathrm{Glc}, \mathrm{R}^{4}=\mathrm{CH}_{3}$

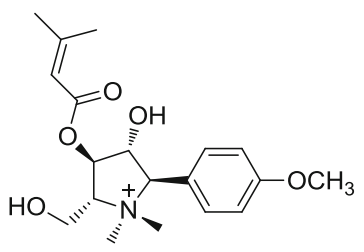

Codonopyrrolidium A (5)

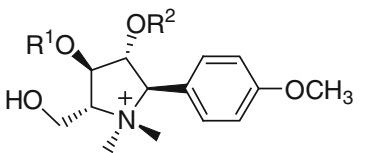

Codonopyrrolidium $B(6): \mathrm{R}^{1}=\mathrm{H}, \mathrm{R}^{2}=\mathrm{H}$ Codotubulosine B (11): $\mathrm{R}^{1}=\mathrm{Ac}, \mathrm{R}^{2}=\mathrm{Ac}$<smiles>[R]c1nccc2c3ccccc3n([R])c12</smiles>

n-9-Formyl harman (12): $\mathrm{R}^{1}=\mathrm{CHO}, \mathrm{R}^{2}=\mathrm{CH}_{3}$ Norharman (13): $\mathrm{R}^{1}=\mathrm{R}^{2}=\mathrm{H}$ 1-Carbomethyl carboline (14): $\mathrm{R}^{1}=\mathrm{H}, \mathrm{R}^{2}=\mathrm{COOCH}_{3}$

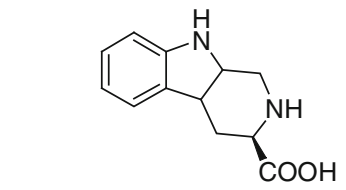

1, 2, 3, 4-Tetrahydro- $\beta$-carboline-3-carboxylic acid (15)<smiles>N[C@@H](Cc1c[nH]c2ccccc12)C(=O)O</smiles>

Tryptophan (16)<smiles>OCc1ccc(-c2nccc3c2[nH]c2ccccc23)o1</smiles>

Perlolyrine (17)

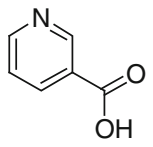

Nicotinic acid (18)

Fig. 1 Structures of alkaloids (1-18) from Codonopsis species

1-yl) phenol (40) and sachaliside (41), were isolated from the roots of $C$. cordifolioidea [28, 29]. Syringin (42) has been commonly found in 5 Codonopsis species [21, 23, 3032].

\section{Triterpenes}

Three new triterpenyl esters, codonopilates A (43), B (44) and $\mathrm{C}$ (45), together with seven known triterpenoids, 24-methylenecycloartanyl linolate (46), 24-methylenecycloartan-3-ol (47), friedelin (48), 1-friedelen-3-one (49), stigmast-7-en-3-one (50), taraxerol (51) and stigmast-7-en3 -ol (52), were isolated from the $\mathrm{CHCl}_{3}$-soluble fraction of the methanol extract of $C$. pilosula [14]. Additionally, $\alpha$ spinasterol (53) was obtained from C. pilosula, C. tangshen, C. lanceolata and C. thalictrifolia [32-35], and taraxeryl acetate (54) was obtained from $C$. pilosula, $C$. tangshen and C. clematidea $[10,34,35]$. The oleanan-type bisdesmoside with sugars at C-3 and C-28, codonolaside (55), codonolasides I (56), II (57) and III (58), and their prosapogenins, eclalbasaponin XIII (59) and echinocystic acid 3-O- $\beta$-D-glucuronopyranoside (60), were isolated from the roots of $C$. lanceolata [36]. The triterpene saponins, lancemasides A (61), B (62), C (63), D (64), E (65), F (66) and $\mathrm{G}(67)$, have also been isolated from the roots of $C$. lanceolata cultivated in Korea [19]. Codonolaside IV (68), codonolaside V (69), foetidissimoside A (70), aster saponin $\mathrm{Hb}$ (71), oleanolic acid (72), echinocystic acid (73) and stigmasterol (74) were found in the roots of $C$. lanceolata
[19, 30, 34, 37-39]. Foetidissimoside A (70) and rubiprasin B (75) were isolated from the aerial parts of C. clematidea [10]. For the aerial parts of $C$. thalictrifolia, isolation of $\alpha$ spinasterol (53) and $\beta$-amyrin acetate (76) was reported [32]. Zeorin (77) and lupeol (78) were isolated from the whole plants of $C$. nervosa [40].

\section{Polyacetylenes}

Isolation and identification of lobetyolin (79), lobetyolinin (80) and lobetyol (81) from the roots and aerial parts of plants belonging to the genus Codonopsis have also been reported [10, 12, 18, 25, 27, 29, 32, 34, 41, 42]. Three new polyacetylene glucosides, cordifolioidynes A $(\mathbf{8 2})$, B (83) and $\mathrm{C}(\mathbf{8 4})$, were isolated from a $95 \%$ ethanol extract of the roots of $C$. cordifolioidea [29]. Recently, cordifolioidynes B (83) has also been found in C. pilosula, $C$. pilosula var. modesta and C. tangshen, which are the botanical sources of Codonopsis Radix [12].

\section{Flavones}

Chrysoeriol (85), tricin (86), wogonin (87) and luteolin (88) were isolated from the roots of $C$. xundianensis Wang $\mathrm{ZT}$ and $\mathrm{Xu}$ GJ, which grows in Yunnan Province, China [43]. Luteolin (88), kaempferol (89), luteolin-5-O- $\beta$-Dglucopyranoside (90), luteolin-7- $O$ - $\beta$-D-gentiobioside (91), apigenin-7-O- $\beta$-D-glucopyranoside (92) and luteolin-7- $O$ - 
Table 1 Compounds in Codonopsis species

\begin{tabular}{|c|c|c|c|c|}
\hline No. & Compound names & Species & Part of the plant & References \\
\hline & Alkaloids & & & \\
\hline 1 & Codonopsine & C. clematidea & Aerial parts & [9] \\
\hline 2 & Codonopsinine & C. clematidea & Aerial parts & [9] \\
\hline 3 & Codonopsinol & C. clematidea & Aerial parts & {$[10]$} \\
\hline \multirow[t]{2}{*}{4} & Radicamine A & C. pilosula & Roots & {$[11]$} \\
\hline & & C. clematidea & Aerial parts & {$[10]$} \\
\hline \multirow[t]{3}{*}{5} & Codonopyrrolidium A & C. pilosula & Roots & {$[12]$} \\
\hline & & $\begin{array}{l}\text { C. pilosula var. } \\
\text { modesta }\end{array}$ & Roots & {$[12]$} \\
\hline & & C. tangshen & Roots & {$[13]$} \\
\hline \multirow[t]{3}{*}{6} & Codonopyrrolidium B & C. pilosula & Roots & [14] \\
\hline & & $\begin{array}{l}\text { C. pilosula var. } \\
\text { modesta }\end{array}$ & Roots & {$[12]$} \\
\hline & & C. tangshen & Roots & {$[13]$} \\
\hline 7 & Codonopsinol A & C. pilosula & Roots & {$[11]$} \\
\hline 8 & Codonopsinol B & C. pilosula & Roots & {$[11]$} \\
\hline 9 & Codonopsinol C & C. pilosula & Roots & {$[11]$} \\
\hline 10 & Codonopiloside A & C. pilosula & Roots & {$[11]$} \\
\hline 11 & Codotubulosine B & C. tubulosa & Roots & {$[15]$} \\
\hline 12 & $n$-9-Formyl harman & C. lanceolata & Roots & [16] \\
\hline 13 & Norharman & C. lanceolata & Roots & [16] \\
\hline 14 & 1-Carbomethyl carboline & C. lanceolata & Roots & {$[16]$} \\
\hline 15 & 1,2,3,4-Tetrahydro- $\beta$-carboline-3-carboxylic acid & C. lanceolata & Roots & {$[17]$} \\
\hline \multirow[t]{2}{*}{16} & Tryptophan & C. pilosula & Roots & {$[18]$} \\
\hline & & C. lanceolata & Roots & [19] \\
\hline 17 & Perlolyrine & C. pilosula & Roots & {$[20]$} \\
\hline 18 & Nicotinic acid & C. pilosula & Roots & {$[21]$} \\
\hline 19 & Uracil & C. pilosula & Roots & {$[18]$} \\
\hline \multirow[t]{3}{*}{20} & Adenosine & C. pilosula & Roots & {$[22]$} \\
\hline & & C. tangshen & Roots & {$[13]$} \\
\hline & Phenylpropanoids & & & \\
\hline \multirow[t]{4}{*}{21} & Tangshenoside I & C. pilosula & Roots & {$[12]$} \\
\hline & & $\begin{array}{l}\text { C. pilosula var. } \\
\text { modesta }\end{array}$ & Roots & {$[12]$} \\
\hline & & C. tangshen & Roots & {$[23]$} \\
\hline & & C. lanceolata & Roots & {$[24]$} \\
\hline \multirow[t]{2}{*}{22} & Tangshenoside II & C. tangshen & Roots & {$[23]$} \\
\hline & & C. lanceolata & Roots & {$[24]$} \\
\hline \multirow[t]{2}{*}{23} & Tangshenoside III & C. tangshen & Roots & {$[25]$} \\
\hline & & C. lanceolata & Roots & {$[24]$} \\
\hline \multirow[t]{2}{*}{24} & Tangshenoside IV & C. tangshen & Roots & {$[25]$} \\
\hline & & C. lanceolata & Roots & {$[24]$} \\
\hline 25 & Tangshenoside V & C. tangshen & Roots & {$[26]$} \\
\hline \multirow[t]{2}{*}{26} & Tangshenoside VI & C. tangshen & Roots & {$[26]$} \\
\hline & & C. nervosa & Aerial parts & {$[27]$} \\
\hline 27 & Codonoside A & C. tangshen & Roots & {$[13]$} \\
\hline 28 & Codonoside B & C. tangshen & Roots & [13] \\
\hline 29 & Tangshenoside VIII & C. lanceolata & Roots & {$[24]$} \\
\hline 30 & Cordifoliketone A & C. cordifolioidea & Roots & {$[28]$} \\
\hline
\end{tabular}


Table 1 continued

\begin{tabular}{|c|c|c|c|c|}
\hline No. & Compound names & Species & Part of the plant & References \\
\hline 31 & Cordifoliketone B & C. cordifolioidea & Roots & {$[28]$} \\
\hline 32 & Coniferaldehyde & C. cordifolioidea & Roots & [29] \\
\hline 33 & Sinapinaldehyde & C. cordifolioidea & Roots & [29] \\
\hline 34 & Coniferoside & C. cordifolioidea & Roots & [29] \\
\hline 35 & Isoconiferin & C. cordifolioidea & Roots & [29] \\
\hline 36 & Nervolan B & C. cordifolioidea & Roots & [28] \\
\hline 37 & Nervolan C & C. cordifolioidea & Roots & [28] \\
\hline 38 & Dillapiole & C. cordifolioidea & Roots & [28] \\
\hline 39 & 1-Allyl-2,6-dimethoxy-3,4-methylenedioxybenzene & C. cordifolioidea & Roots & [28] \\
\hline 40 & 4-Allyl-2-(3-methylbut-2-en-1-yl) phenol & C. cordifolioidea & Roots & [28] \\
\hline 41 & Sachaliside & C. cordifolioidea & Roots & [29] \\
\hline \multirow[t]{6}{*}{42} & \multirow[t]{5}{*}{ Syringin } & C. pilosula & Roots & [21] \\
\hline & & C. tangshen & Roots & [23] \\
\hline & & C. lanceolata & Roots & {$[30]$} \\
\hline & & C. nervosa & Aerial parts & [31] \\
\hline & & C. thalictrifolia & Aerial parts & [32] \\
\hline & \multicolumn{4}{|l|}{ Triterpenes } \\
\hline 43 & Codonopilate A & C. pilosula & Roots & [14] \\
\hline 44 & Codonopilate B & C. pilosula & Roots & {$[14]$} \\
\hline 45 & Codonopilate $\mathrm{C}$ & C. pilosula & Roots & {$[14]$} \\
\hline 46 & 24-Methylenecycloartanyl linolate & C. pilosula & Roots & [14] \\
\hline 47 & 24-Methylenecycloartan-3-ol & C. pilosula & Roots & [14] \\
\hline \multirow[t]{3}{*}{48} & \multirow[t]{3}{*}{ Friedelin } & C. pilosula & Roots & [14] \\
\hline & & C. tangshen & Roots & [12] \\
\hline & & C. lanceolata & Roots & [33] \\
\hline 49 & 1-Friedelen-3-one & C. pilosula & Roots & {$[14]$} \\
\hline 50 & Stigmast-7-en-3-one & C. pilosula & Roots & {$[14]$} \\
\hline \multirow[t]{2}{*}{51} & \multirow[t]{2}{*}{ Taraxerol } & C. pilosula & Roots & [14] \\
\hline & & C. tangshen & Roots & {$[34]$} \\
\hline 52 & Stigmast-7-en-3-ol & C. pilosula & Roots & {$[14]$} \\
\hline \multirow[t]{4}{*}{53} & \multirow[t]{4}{*}{$\alpha$-Spinasterol } & C. pilosula & Roots & {$[35]$} \\
\hline & & C. tangshen & Roots & {$[34]$} \\
\hline & & C. lanceolata & Roots & [33] \\
\hline & & C. thalictrifolia & Aerial parts & {$[32]$} \\
\hline \multirow[t]{3}{*}{54} & \multirow[t]{3}{*}{ Taraxeryl acetate } & C. pilosula & Roots & [35] \\
\hline & & C. tangshen & Roots & [34] \\
\hline & & C. clematidea & Aerial parts & [10] \\
\hline 55 & Codonolaside & C. lanceolata & Roots & [36] \\
\hline 56 & Codonolaside I & C. lanceolata & Roots & [36] \\
\hline 57 & Codonolaside II & C. lanceolata & Roots & [36] \\
\hline 58 & Codonolaside III & C. lanceolata & Roots & [36] \\
\hline 59 & Eclalbasaponin XIII & C. lanceolata & Roots & [36] \\
\hline 60 & Echinocystic acid-3-O-(6'-O-methyl)- $\beta$-D-glucuronopyranoside & C. lanceolata & Roots & [36] \\
\hline 61 & Lancemaside A & C. lanceolata & Roots & [19] \\
\hline 62 & Lancemaside B & C. lanceolata & Roots & [19] \\
\hline 63 & Lancemaside C & C. lanceolata & Roots & [19] \\
\hline 64 & Lancemaside D & C. lanceolata & Roots & [19] \\
\hline 65 & Lancemaside E & C. lanceolata & Roots & [19] \\
\hline
\end{tabular}


Table 1 continued

\begin{tabular}{|c|c|c|c|c|}
\hline No. & Compound names & Species & Part of the plant & References \\
\hline 66 & Lancemaside F & C. lanceolata & Roots & [19] \\
\hline 67 & Lancemaside $\mathrm{G}$ & C. lanceolata & Roots & [19] \\
\hline 68 & Codonolaside IV & C. lanceolata & Roots & {$[37]$} \\
\hline 69 & Codonolaside V & C. lanceolata & Roots & {$[38]$} \\
\hline \multirow[t]{2}{*}{70} & Foetidissimoside A & C. lanceolata & Roots & [19] \\
\hline & & C. clematidea & Aerial parts & {$[10]$} \\
\hline 71 & Aster saponin $\mathrm{Hb}$ & C. lanceolata & Roots & [39] \\
\hline 72 & Oleanolic acid & C. lanceolata & Roots & {$[30]$} \\
\hline 73 & Echinocystic acid & C. lanceolata & Roots & {$[30]$} \\
\hline \multirow[t]{2}{*}{74} & Stigmasterol & C. tangshen & Roots & {$[34]$} \\
\hline & & C. lanceolata & Roots & {$[33]$} \\
\hline 75 & Rubiprasin B & C. clematidea & Aerial parts & {$[10]$} \\
\hline 76 & $\beta$-Amyrin acetate & C. thalictrifolia & Aerial parts & {$[32]$} \\
\hline 77 & Zeorin & C. nervosa & Whole plants & [40] \\
\hline \multirow[t]{2}{*}{78} & Lupeol & C. nervosa & Whole plants & [40] \\
\hline & Polyacetylenes & & & \\
\hline \multirow[t]{8}{*}{79} & Lobetyolin & Codonopsis pilosula & Roots & {$[18]$} \\
\hline & & $\begin{array}{l}\text { C. pilosula var. } \\
\text { modesta }\end{array}$ & Roots & [12] \\
\hline & & C. tangshen & Roots & {$[25]$} \\
\hline & & C. lanceolata & Roots & [41] \\
\hline & & C. clematidea & Aerial parts & {$[10]$} \\
\hline & & C. cordifolioidea & Roots & [29] \\
\hline & & C. nervosa & Whole plants & {$[27]$} \\
\hline & & C. thalictrifolia & Aerial parts & {$[32]$} \\
\hline \multirow[t]{4}{*}{80} & Lobetyolinin & C. pilosula & Roots & [18] \\
\hline & & $\begin{array}{l}\text { C. pilosula var. } \\
\text { modesta }\end{array}$ & Roots & {$[12]$} \\
\hline & & C. tangshen & Roots & {$[12]$} \\
\hline & & C. clematidea & Aerial parts & [10] \\
\hline \multirow[t]{4}{*}{81} & Lobetyol & C. pilosula & Roots & [42] \\
\hline & & $\begin{array}{l}\text { C. pilosula var. } \\
\text { modesta }\end{array}$ & Roots & {$[12]$} \\
\hline & & C. tangshen & Roots & {$[34]$} \\
\hline & & C. cordifolioidea & Roots & [29] \\
\hline 82 & Cordifolioidyne A & C. cordifolioidea & Roots & [29] \\
\hline \multirow[t]{4}{*}{83} & Cordifolioidyne B & C. pilosula & Roots & {$[12]$} \\
\hline & & $\begin{array}{l}\text { C. pilosula var. } \\
\text { modesta }\end{array}$ & Roots & {$[12]$} \\
\hline & & C. tangshen & Roots & [12] \\
\hline & & C. cordifolioidea & Roots & [29] \\
\hline \multirow[t]{2}{*}{84} & Cordifolioidyne C & C. cordifolioidea & Roots & [29] \\
\hline & Flavones & & & \\
\hline 85 & Chrysoeriol & C. xundianensis & Roots & {$[43]$} \\
\hline 86 & Tricin & C. xundianensis & Roots & [43] \\
\hline 87 & Wogonin & C. xundianensis & Roots & [43] \\
\hline
\end{tabular}


Table 1 continued

\begin{tabular}{|c|c|c|c|c|}
\hline No. & Compound names & Species & Part of the plant & References \\
\hline \multirow[t]{4}{*}{88} & \multirow[t]{4}{*}{ Luteolin } & C. nervosa & Whole plants & {$[40]$} \\
\hline & & C. thalictrifolia & Aerial parts & {$[32]$} \\
\hline & & C. clematidea & Aerial parts & {$[10]$} \\
\hline & & C. xundianensis & Roots & [43] \\
\hline 89 & Kaempferol & C. nervosa & Whole plants & {$[40]$} \\
\hline \multirow[t]{2}{*}{90} & \multirow[t]{2}{*}{ Luteolin-5- $O$ - $\beta$-D-glucopyranoside } & C. nervosa & Aerial parts & [27] \\
\hline & & C. thalictrifolia & Aerial parts & [32] \\
\hline \multirow[t]{2}{*}{91} & \multirow[t]{2}{*}{ Luteolin-7- $O$ - $\beta$-D-gentiobioside } & C. nervosa & Aerial parts & [27] \\
\hline & & C. thalictrifolia & Aerial parts & {$[32]$} \\
\hline 92 & Apigenin-7- $O-\beta$-D-glucopyranoside & C. nervosa & Aerial parts & {$[31]$} \\
\hline \multirow[t]{2}{*}{93} & \multirow{2}{*}{$\begin{array}{l}\text { Luteolin-7- } O \text { - } \beta \text {-D-glucopyranosyl }(1 \rightarrow 6)-\left[\left(6^{\prime \prime \prime}-O \text {-caffeoyl }\right)\right]-\beta \text {-D- } \\
\text { glucopyranoside }\end{array}$} & C. nervosa & Whole plants & {$[40]$} \\
\hline & & C. clematidea & Aerial parts & {$[10]$} \\
\hline \multirow[t]{2}{*}{94} & Hesperidin & C. pilosula & Roots & {$[35]$} \\
\hline & Organic acids & & & \\
\hline \multirow[t]{2}{*}{95} & Succinic acid & C. pilosula & Roots & [44] \\
\hline & & C. nervosa & Aerial parts & {$[31]$} \\
\hline \multirow[t]{2}{*}{96} & 3-O-caffeoylquinic acid & C. nervosa & Aerial parts & {$[31]$} \\
\hline & & C. thalictrifolia & Aerial parts & {$[32]$} \\
\hline 97 & 5-O-caffeoylquinic acid & C. nervosa & Aerial parts & {$[31]$} \\
\hline 98 & 4-( $\beta$-D-Glucopyranosyl)-benzoic acid & C. nervosa & Aerial parts & {$[31]$} \\
\hline 99 & Caffeic acid & C. thalictrifolia & Aerial parts & {$[32]$} \\
\hline 100 & Linoleic acid & C. thalictrifolia & Aerial parts & {$[32]$} \\
\hline 101 & 9,10,13-Trihydroxy-(E)-octadec-11-enoic acid & C. pilosula & Roots & {$[35]$} \\
\hline 102 & Shikimic acid & C. lanceolata & Roots & {$[33]$} \\
\hline \multirow[t]{2}{*}{103} & Vanillic acid & C. tangshen & Roots & {$[34]$} \\
\hline & Other compounds & & & \\
\hline 104 & Atractylenolide III & C. pilosula & Roots & {$[21]$} \\
\hline \multirow[t]{2}{*}{105} & 5-Hydroxymethyl-2-furaldehyde & C. pilosula & Roots & {$[14]$} \\
\hline & & C. tangshen & Roots & {$[34]$} \\
\hline 106 & Angelicin & C. pilosula & Roots & [44] \\
\hline 107 & Psoralen & C. pilosula & Roots & {$[44]$} \\
\hline 108 & Emodin & C. pilosula & Roots & {$[18]$} \\
\hline 109 & Geniposide & C. pilosula & Roots & {$[17]$} \\
\hline 110 & Hexyl- $\beta$-D-glucopyranoside & C. pilosula & Roots & {$[18]$} \\
\hline 111 & Butyl- $\beta$-D-fructournanoside & C. pilosula & Roots & {$[18]$} \\
\hline \multirow[t]{2}{*}{112} & $\beta$-Sitosterol & C. pilosula & Roots & {$[35]$} \\
\hline & & C. nervosa & Whole plants & {$[40]$} \\
\hline 113 & $\beta$-Daucosterol & C. pilosula & Roots & {$[35]$} \\
\hline 114 & Hexyl- $\beta$-gentiobioside & C. tangshen & Roots & {$[25]$} \\
\hline 115 & Hexyl- $\beta$-sophoroside & C. tangshen & Roots & {$[25]$} \\
\hline 116 & $(E)$-2-hexenyl- $\beta$-sophoroside & C. tangshen & Roots & {$[25]$} \\
\hline \multirow[t]{2}{*}{117} & (E)-2-hexenyl- $\alpha$-L-arabinopyranosyl( $1 \rightarrow 6)$ - $\beta$-D-glucopyranoside & C. tangshen & Roots & {$[25]$} \\
\hline & & C. clematidea & Aerial parts & {$[10]$} \\
\hline 118 & Cordifoliflavane A & C. cordifolioidea & Roots & [45] \\
\hline 119 & Cordifoliflavane B & C. cordifolioidea & Roots & [45] \\
\hline 120 & Lanceolune A & C. lanceolata & Roots & [46] \\
\hline 121 & Lanceolune B & C. lanceolata & Roots & [46] \\
\hline 122 & Lanceolune $\mathrm{C}$ & C. lanceolata & Roots & [46] \\
\hline
\end{tabular}


Table 1 continued

\begin{tabular}{|c|c|c|c|c|}
\hline No. & Compound names & Species & Part of the plant & References \\
\hline 123 & Codonocerebroside A & C. lanceolata & Roots & [41] \\
\hline 124 & 3-Oxo- $\alpha$-ionol- $\beta$-D-glucopyranoside & C. clematidea & Aerial parts & {$[10]$} \\
\hline 125 & 1,6-Hexanediol,3,4-bis(4-hydroxy-3-methoxyphenyl) & C. clematidea & Aerial parts & {$[10]$} \\
\hline 126 & Sweroside & C. nervosa & Whole plants & [40] \\
\hline
\end{tabular}

$\beta$-D-glucopyranosyl( $1 \rightarrow 6)$ - $\left[\left(6^{\prime \prime \prime}-O\right.\right.$-caffeoyl $\left.)\right]-\beta$-D-glucopyranoside (93) were obtained from $C$. nervosa [27, 31, 40], and luteolin (88), luteolin-5-O- $\beta$-D-glucopyranoside (90) and luteolin-7- $O$ - $\beta$-D-gentiobioside (91) were also found in the aerial parts of $C$. thalictrifolia [32]. In addition, luteolin $(\mathbf{8 8})$ and luteolin-7-O- $\beta$-D-glucopyrano$\operatorname{syl}(1 \rightarrow 6)-\left[\left(6^{\prime \prime \prime}-O\right.\right.$-caffeoyl $\left.)\right]-\beta$-D-glucopyranoside were isolated from the aerial parts of $C$. clematidea [10]. Hesperidin (94) was only isolated from the roots of $C$. pilosula [35].

\section{Organic acids}

To date, succinic acid (95), 3- $O$-caffeoylquinic acid (96), 5-O-caffeoylquinic acid (97) and 4-( $\beta$-D-glucopyranosyl)benzoic acid (98) have been found in $C$. nervosa [31]. Caffeic acid (99), linoleic acid (100) and 3-O-caffeoylquinic acid (96) were isolated from $C$. thalictrifolia [32]. Succinic acid (95) and 9,10,13-trihydroxy-(E)-octadec-11enoic acid (101) were isolated from C. pilosula [35, 44]. Shikimic acid (102) and vanillic acid (103) were only obtained from the roots of $C$. lanceolata and $C$. tangshen, respectively [33, 34].

\section{Other compounds}

Atractylenolide III (104), 5-hydroxymethyl-2-furaldehyde (105), angelicin (106), psoralen (107), emodin (108), geniposide (109), hexyl- $\beta$-D-glucopyranoside (110), butyl- $\beta$ D-fructournanoside (111), $\beta$-sitosterol (112) and $\beta$-daucosterol (113) were isolated from the roots of $C$. pilosula $[14,17,18,21,35,44]$. Hexyl- $\beta$-gentiobioside (114), hexyl- $\beta$-sophoroside (115), (E)-2-hexenyl- $\beta$-sophoroside (116), (E)-2-hexenyl- $\alpha$-L-arabinopyranosyl $(1 \rightarrow 6)-\beta$-Dglucopyranoside (117) and 5-hydroxymethyl-2-furaldehyde (105) were isolated from the roots of $C$. tangshen $[25,34]$. Cordifoliflavanes A (118) and B (119) were isolated from the roots of $C$. cordifolioidea [45]. Three new benzofuranylpropanoids, lanceolunes A (120), B (121) and $\mathrm{C}$ (122), as well as a new cerebroside, codonocerebroside A (123), have been isolated from the roots of $C$. lanceolata $[41,46]$. (E)-2-Hexenyl- $\alpha$-L-arabinopyranosyl $(1 \rightarrow 6)-\beta$-D- glucopyranoside (117), 3-oxo- $\alpha$-ionol- $\beta$-D-glucopyranoside (124) and 1,6-hexanediol,3,4-bis(4-hydroxy-3-methoxyphenyl) (125) were isolated from the aerial parts of $C$. clematidea [10]. In addition, sweroside (126) and $\beta$-sitosterol (112) were obtained from the whole plants of $C$. nervosa [40]. Nutritive constituents including amino acids and trace elements in $C$. pilosula have been reported [47].

\section{Essential oils}

As one of the important compositions, essential oils of several Codonopsis species have been reported. In the essential components from $C$. pilosula, 50 of 66 separated components were identified by GC-MS, mainly containing 1,2-benzonedicarboxylic acid dibatyl-ester (12.45\%), heptedecanoic acid (8.10\%) and 2,4,5-triisopropyl styrene (7.62\%) [48]. Using the GC-MS method, 54 peaks were separated and 37 of them were identified in the essential components extracted from $C$. clematidea, in which the most abundant component was methyl hexadecanoate $(30.40 \%)$ [49]. The essential oils from the whole plants of C. thalictrifolia, as a traditional Tibetan medicine, were analyzed by GC-MS, and 45 of 60 separated components were identified by comparing their mass spectra, in which the main principles were palmitic acid (43.5\%) and linolic acid $(18.3 \%)$ [50]. In the essential oils extracted from the fresh and dried roots of $C$. cordifolioidea, 63 compounds were identified by GC-MS analysis, indicating that linolic acid $(21.9 \%)$, retene $(11.4 \%)$, pentadecane $(7.4 \%)$, methyl 9,12,15-octadecatrienoate $(6.8 \%)$ and heneicosylcyclopentane $(3.8 \%)$ were the main components [51].

\section{Polysaccharides}

Large-molecule components in Codonopsis species were also studied. A water-soluble polysaccharide with a molecular mass of $1.1 \times 10^{4} \mathrm{Da}$ was obtained from the roots of $C$. pilosula and its structure investigation revealed that this polysaccharide had a backbone consisting of $(1 \rightarrow 3)$-linked- $\beta$-D-galactopyranosyl, $(1 \rightarrow 2,3)$-linked$\alpha$-D-galactopyranosyl and $(1 \rightarrow 3)$-linked- $\beta$-D-rhamnopyranosyl residues and were branched with two glycosyl 


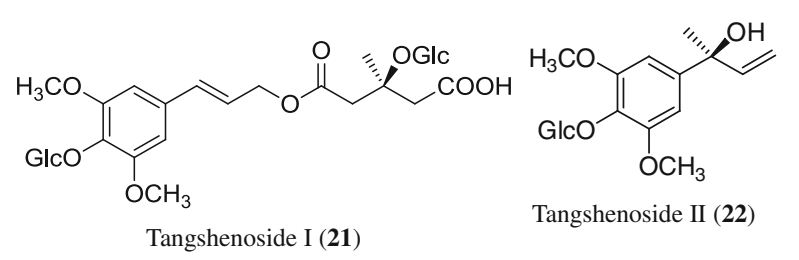<smiles>COc1cc(/C=C/COC(=O)C[C@](C)(CC(=O)O)OC(=O)O)cc(/C=C/Cc2c(OC)c(OC)c(OC)c(OC)c2OC)c1</smiles>

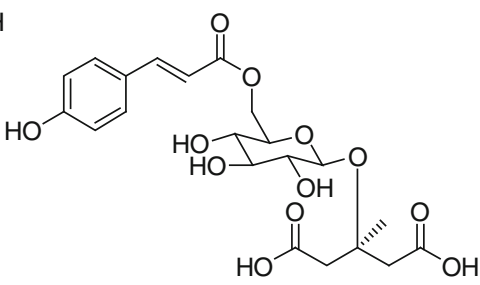
Tangshenoside V (25)<smiles>COc1cc(/C=C/Cc2c(/C=C/CO)cc(OC)c(OC)c2OC)cc(OC)c1OC(C)(O)O</smiles>

Tangshenoside III (23)<smiles></smiles><smiles>COc1cc(/C=C/COC(=O)CC(C)(CC(=O)O)OC2OC(COC(=O)/C=C/c3ccc(O)cc3)C(O)C(O)C2O)cc(OC)c1OC(Cl)Cl</smiles>

Codonoside A (27)<smiles>COc1cc(/C=C/COC(=O)CC(C)(CC(=O)O)OOC(O)C(COC(=O)/C=C\c2ccc(O)cc2)C(O)C(O)CO)cc(OC)c1OC</smiles>

Codonoside B (28)<smiles>COc1cc(/C=C/COC(=O)CC(C)(O)CC(=O)OC/C=C/c2cc(OC)c(OC)c(OC)c2)cc(OC)c1OC</smiles>

Tangshenoside VIII (29)<smiles>[R]c1cc(/C=C/C[Tl])cc([R])c1[R]</smiles>

Coniferoside (34): $\mathrm{R}^{1}=\mathrm{OH}, \mathrm{R}^{2}=\mathrm{OCH}_{3}, \mathrm{R}^{3}=\mathrm{O}-\mathrm{Glc}, \mathrm{R}^{4}=\mathrm{H}$ Isoconiferin (35): : : $\mathrm{R}^{1}=\mathrm{O}-\mathrm{Glc}, \mathrm{R}^{2}=\mathrm{OCH}_{3}, \mathrm{R}^{3}=\mathrm{OH}, \mathrm{R}^{4}=\mathrm{H}$ Sachaliside (41 ): $\mathrm{R}^{1}=\mathrm{O}-\mathrm{Glc}, \mathrm{R}^{2}=\mathrm{H}, \mathrm{R}^{3}=\mathrm{OH}, \mathrm{R}^{4}=\mathrm{H}$ Syringin (42): $\mathrm{R}^{1}=\mathrm{OH}, \mathrm{R}^{2}=\mathrm{OCH}_{3}, \mathrm{R}^{3}=\mathrm{O}-\mathrm{Glc}, \mathrm{R}^{4}=\mathrm{OCH}_{3}$<smiles>[R7]c1c(CC=C)c(OC)c([R7])c2c1OCO2</smiles>

Dillapiole (38): $\mathrm{R}^{1}=\mathrm{H}, \mathrm{R}^{2}=\mathrm{OCH}_{3}$

1-Allyl-2,6-dimethoxy-3,4-methylenedioxybenzene (39): $\mathrm{R}^{1}=\mathrm{OCH}_{3}, \mathrm{R}^{2}=\mathrm{H}$<smiles>COc1cc(O)c(C(=O)CCO)cc1OC</smiles>

Cordifoliketone A (30): $\mathrm{R}=\mathrm{Ac}$<smiles>[R]c1cc(CCCO)cc(OC)c1O[Na]</smiles>

Nervolan B (36): $\mathrm{R}=\mathrm{OCH}_{3}$ Nervolan C (37): R=H<smiles>C=CCc1ccc(O)c(CC=C(C)C)c1</smiles>

4-Allyl-2-(3-methylbut-2-en-1-yl) phenol (40)

Fig. 2 Structures of phenylpropanoids (21-42) from Codonopsis species

residues composed of $\alpha$-L-arabinose- $(1 \rightarrow 5)$ - $\alpha$-L-arabinose, whose $\mathrm{C}-1$ linked residues at the $O-2$ position of galactosyl along the main chain in the ratio of 1:1:2:1:1
[52]. Another polysaccharide with a molecular mass of $7.4 \times 10^{4} \mathrm{Da}$ was isolated from C. pilosula and its components were galactose, arabinose and rhamnose in the 


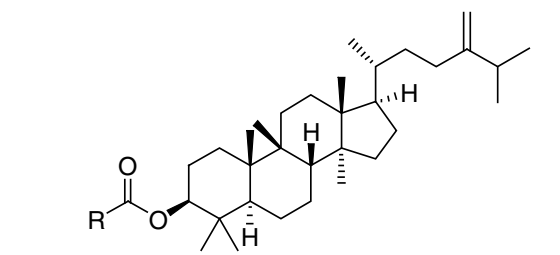

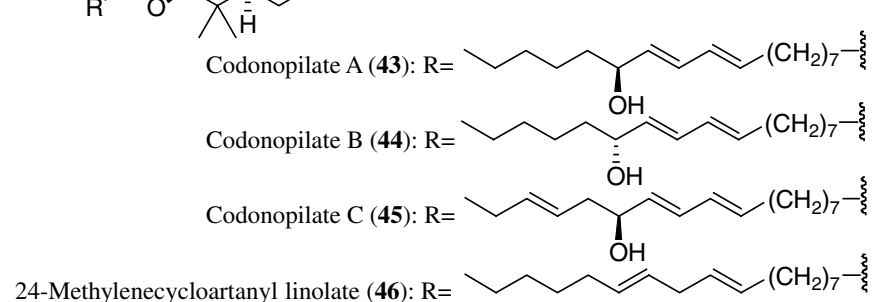

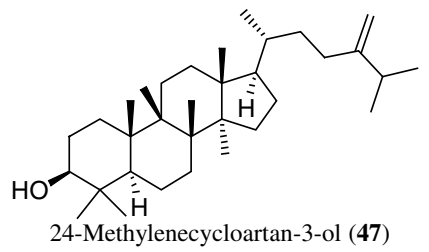<smiles>C[C@H]1C(=O)CC[C@]2(C)[C@@H]1[C@]1(C)CC[C@@]3(C)[C@@H](CC[C@]12C)[C@@]1(C)CC(C)(C)CC[C@]31C</smiles>

Friedelin (48)

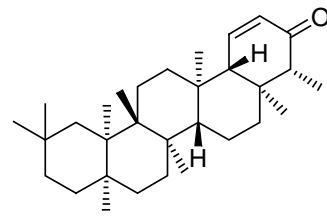

1-Friedelen-3-one (49)<smiles>CCC(CC[C@@H](C)C1CCC2C3=CCCCC3CCC21C)C(C)C</smiles>

Stigmast-7-en-3-one (50)

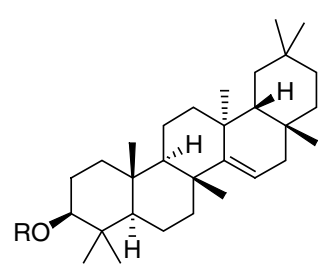

Taraxerol (51): $\mathrm{R}=\mathrm{H}$

Taraxeryl acetate $(\mathbf{5 4})$ : $\mathrm{R}=\mathrm{Ac}$

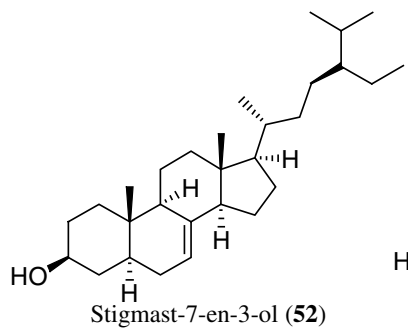<smiles>C1CCCCC1</smiles><smiles>CC(C)C1CCC2C3=CCC4CCCCC4(C)C3CCC21C</smiles>

$\alpha$-Spinasterol (53)<smiles>C=CC(CC)C(C)C</smiles><smiles>CC1(C)CCC2(C(=O)O)CCC34CCCCC3CCC4CCC23CCCCC13</smiles>
Oleanolic acid (72)<smiles>CC1(C(=O)O)CC[C@@]2(C)C(=CCC3[C@@]4(C)CC[C@@H](O)C(C)(O)C4CC[C@]32C)C1</smiles>

$\mathrm{HO}$<smiles>CC[C@H](/C=C/[C@@H](C)[C@H]1CC[C@H]2C3CC=C4CC(O)CC[C@]4(C)[C@H]3CC[C@]21C)C(C)C</smiles>

Stigmasterol (74)

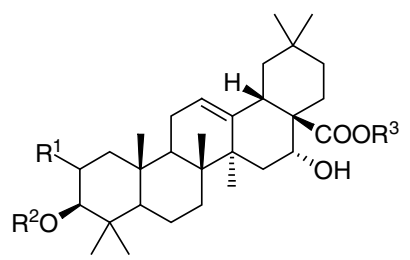

Codonolaside (55): $\mathrm{R}^{1}=\mathrm{H}, \mathrm{R}^{2}=\mathrm{Xyl}(1 \rightarrow 3)$-GlcA, $\mathrm{R}^{3}=\mathrm{Xyl}(1 \rightarrow 4)$ - $\mathrm{Rha}(1 \rightarrow 2)$-Ara

Codonolaside I (56): $\mathrm{R}^{1}=\mathrm{H}, \mathrm{R}^{2}=\mathrm{Xyl}(1 \rightarrow 3)\left(6-\mathrm{O}-\right.$-methyl)-GlcA, $\mathrm{R}^{3}=\mathrm{Xyl}(1 \rightarrow 4)-\mathrm{Rha}(1 \rightarrow 2)$-Ara

Codonolaside II (57): $\mathrm{R}^{1}=\mathrm{H}, \mathrm{R}^{2}=\mathrm{H}, \mathrm{R}^{3}=\mathrm{Xyl}(1 \rightarrow 4)-\mathrm{Rha}(1 \rightarrow 2)$-Ara

Codonolaside III (58): $\mathrm{R}^{1}=\mathrm{H}, \mathrm{R}^{2}=\mathrm{Xyl}(1 \rightarrow 3)$-GlcA, $\mathrm{R}^{3}=\mathrm{Xyl}(1 \rightarrow 4)$-Rha $(1 \rightarrow 2)[\mathrm{Glc}(1 \rightarrow 4)]$-Ara Eclalbasaponin XIII (59): $\mathrm{R}^{1}=\mathrm{H}, \mathrm{R}^{2}=\left(6-\mathrm{O}-\right.$-methyl)-GlcA, $\mathrm{R}^{3}=\mathrm{H}$

Echinocystic acid-3-O-(6'-O-methyl) $\beta$-D-glucuronopyranoside $(\mathbf{6 0}): \mathrm{R}^{1}=\mathrm{H}, \mathrm{R}^{2}=\mathrm{GlcA}, \mathrm{R}^{3}=\mathrm{H}$

Lancemaside A (61): $\mathrm{R}^{1}=\mathrm{H}, \mathrm{R}^{2}=\mathrm{GlcA}, \mathrm{R}^{3}=\mathrm{Xyl}(1 \rightarrow 3)$-Xyl $(1 \rightarrow 4)-\mathrm{Rha}(1 \rightarrow 2)$-Ara

Lancemaside B (62): $\mathrm{R}^{1}=\mathrm{H}, \mathrm{R}^{2}=\mathrm{GlcA}, \mathrm{R}^{3}=\mathrm{Xyl}(1 \rightarrow 3)$-Xyl $(1 \rightarrow 4)-[(1 \rightarrow 3)$-Glc $]-\mathrm{Rha}(1 \rightarrow 2)$-Ara

Lancemaside C (63): $\mathrm{R}^{1}=\mathrm{H}, \mathrm{R}^{2}=\mathrm{GlcA}, \mathrm{R}^{3}=\mathrm{Xyl}(1 \rightarrow 4)-\mathrm{Rha}(1 \rightarrow 2)-[(1 \rightarrow 3)$-Glc]-Ara

Lancemaside D $(\mathbf{6 4}): \mathrm{R}^{1}=\mathrm{H}, \mathrm{R}^{2}=\mathrm{GlcA}, \mathrm{R}^{3}=\mathrm{Rha}(1 \rightarrow 2)-[(1 \rightarrow 3)$-Glc $]$-Ara

Lancemaside E (65): $\mathrm{R}^{1}=\mathrm{H}, \mathrm{R}^{2}=\mathrm{Glc}(1 \rightarrow 3)$-GlcA, $\mathrm{R}^{3}=\mathrm{Xyl}(1 \rightarrow 3)$-Xyl $(1 \rightarrow 4)$-Rha( $(1 \rightarrow 2)$-Ara

Lancemaside $\mathrm{F}(\mathbf{6 6}): \mathrm{R}^{1}=\mathrm{H}, \mathrm{R}^{2}=\mathrm{Glc}(1 \rightarrow 3)-G l c A, \mathrm{R}^{3}=\operatorname{Xyl}(1 \rightarrow 3)-\mathrm{Xyl}(1 \rightarrow 4)-\mathrm{Rha}(1 \rightarrow 2)-[(1 \rightarrow 3)$-Glc $]$-Ara

Lancemaside G (67): $\mathrm{R}^{1}=\mathrm{OH}, \mathrm{R}^{2}=\mathrm{GlcA}, \mathrm{R}^{3}=\mathrm{Xyl}(1 \rightarrow 3)$-Xyl $(1 \rightarrow 4)$-Rha( $(1 \rightarrow 2)$-Ara

Codonolaside IV $(\mathbf{6 8}): \mathrm{R}^{1}=\mathrm{H}, \mathrm{R}^{2}=\mathrm{GlcA}(1 \rightarrow 3)-\mathrm{Xyl}, \mathrm{R}^{3}=\mathrm{Xyl}(1 \rightarrow 4)-\mathrm{Rha}(1 \rightarrow 2)$-Ara

Codonolaside V $(\mathbf{6 9}): \mathrm{R}^{1}=\mathrm{H}, \mathrm{R}^{2}=\mathrm{GlcA}(1 \rightarrow 3)\left(6-\mathrm{O}-\right.$ butyl)-Xyl, $\mathrm{R}^{3}=\mathrm{Xyl}(1 \rightarrow 4)$-Rha $(1 \rightarrow 2)$-Ara

Foetidissimoside A (70): $\mathrm{R}^{1}=\mathrm{H}, \mathrm{R}^{2}=\mathrm{GlcA}, \mathrm{R}^{3}=\mathrm{Xyl}(1 \rightarrow 4)$ - $\mathrm{Rha}(1 \rightarrow 2)$-Ara

Aster saponin $\mathrm{Hb}(\mathbf{7 1}): \mathrm{R}^{1}=\mathrm{H}, \mathrm{R}^{2}=\mathrm{GlcA}, \mathrm{R}^{3}=\mathrm{Rha}(1 \rightarrow 2)$-Ara

Echinocystic acid (73): $\mathrm{R}^{1}=\mathrm{R}^{2}=\mathrm{R}^{3}=\mathrm{H}$

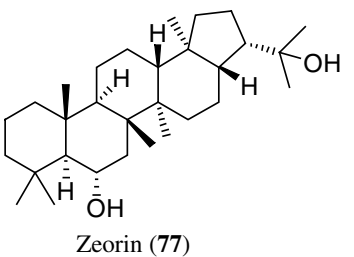

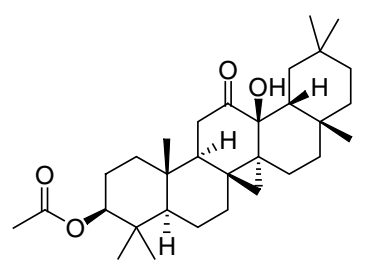

Rubiprasin B (75)<smiles>CC(=O)O[C@H]1CC[C@]2(C)[C@@H]3CC=C4[C@@]5(C)CC(C)(C)CC[C@]5(C)CC[C@]4(C)[C@H]3CC[C@]2(C)C1C</smiles>

$\beta$-Amyrin acetate (76)<smiles>C=C(C)[C@H]1CCC2(C)CC[C@]3(C)[C@H](CC[C@@H]4[C@@]5(C)CC[C@H](O)C(C)(C)[C@@H]5CC[C@]43C)[C@@H]12</smiles>

Lupeol (78)

Fig. 3 Structures of triterpenes (43-78) from Codonopsis species 
Fig. 4 Structures of polyacetylenes (79-84) from Codonopsis species

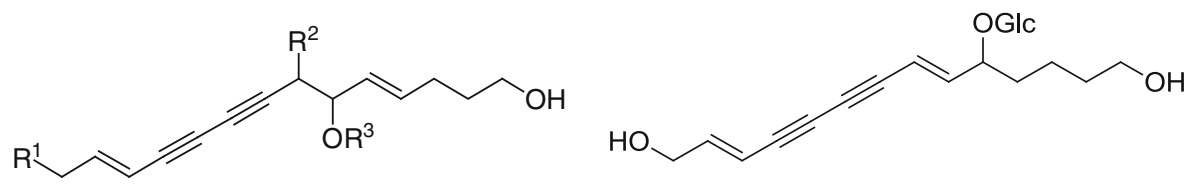

Lobetyolin (79): $\mathrm{R}^{1}=\mathrm{H}, \mathrm{R}^{2}=\mathrm{OH}, \mathrm{R}^{3}=\mathrm{Glc}$

Lobetyolinin $(\mathbf{8 0})$ : $\mathrm{R}^{1}=\mathrm{H}, \mathrm{R}^{2}=\mathrm{OH}, \mathrm{R}^{3}=\mathrm{Glc}(1 \rightarrow 6)-\mathrm{Glc}$

Cordifolioidyne B (83)

Lobetyol (81): $\mathrm{R}^{1}=\mathrm{H}, \mathrm{R}^{2}=\mathrm{OH}, \mathrm{R}^{3}=\mathrm{H}$

Cordifolioidyne A (82): $\mathrm{R}^{1}=\mathrm{R}^{2}=\mathrm{OH}, \mathrm{R}^{3}=\mathrm{Glc}$

Cordifolioidyne $\mathrm{C}(\mathbf{8 4}): \mathrm{R}^{1}=\mathrm{R}^{2}=\mathrm{H}, \mathrm{R}^{3}=\mathrm{Glc}$<smiles></smiles>

Chrysoeriol (85): $\mathrm{R}^{1}=\mathrm{R}^{4}=\mathrm{R}^{7}=\mathrm{H}, \mathrm{R}^{2}=\mathrm{R}^{3}=\mathrm{R}^{6}=\mathrm{OH}, \mathrm{R}^{5}=\mathrm{OCH}_{3}$

Tricin (86): $\mathrm{R}^{1}=\mathrm{R}^{4}=\mathrm{H}, \mathrm{R}^{2}=\mathrm{R}^{3}=\mathrm{R}^{6}=\mathrm{OH}, \mathrm{R}^{5}=\mathrm{R}^{7}=\mathrm{OCH}_{3}$

Wogonin (87): $\mathrm{R}^{1}=\mathrm{R}^{5}=\mathrm{R}^{6}=\mathrm{R}^{7}=\mathrm{H}, \mathrm{R}^{2}=\mathrm{R}^{3}=\mathrm{OH}, \mathrm{R}^{4}=\mathrm{OCH}_{3}$

Luteolin (88): $\mathrm{R}^{1}=\mathrm{R}^{4}=\mathrm{R}^{7}=\mathrm{H}, \mathrm{R}^{2}=\mathrm{R}^{3}=\mathrm{R}^{5}=\mathrm{R}^{6}=\mathrm{OH}$

Kaempferol (89): $\mathrm{R}^{1}=\mathrm{R}^{2}=\mathrm{R}^{3}=\mathrm{OH}, \mathrm{R}^{4}=\mathrm{R}^{5}=\mathrm{R}^{7}=\mathrm{H}, \mathrm{R}^{6}=\mathrm{OH}$

Luteolin-5-O- $\beta$-D-glucopyranoside (90): $\mathrm{R}^{1}=\mathrm{R}^{4}=\mathrm{R}^{7}=\mathrm{H}, \mathrm{R}^{2}=\mathrm{O}-\mathrm{Glc}, \mathrm{R}^{3}=\mathrm{R}^{5}=\mathrm{R}^{6}=\mathrm{OH}$

Luteolin-7-O- $\beta$-D-gentiobioside (91): $\mathrm{R}^{1}=\mathrm{R}^{4}=\mathrm{R}^{7}=\mathrm{H}, \mathrm{R}^{2}=\mathrm{OH}, \mathrm{R}^{3}=\mathrm{O}-\mathrm{Glc}(6 \rightarrow 1)-\mathrm{Glc}, \mathrm{R}^{5}=\mathrm{R}^{6}=\mathrm{OH}$

Apigenin-7-O- $\beta$-D-glucopyranoside (92): $\mathrm{R}^{1}=\mathrm{R}^{4}=\mathrm{R}^{5}=\mathrm{R}^{7}=\mathrm{H}, \mathrm{R}^{2}=\mathrm{R}^{3}=\mathrm{R}^{6}=\mathrm{OH}$

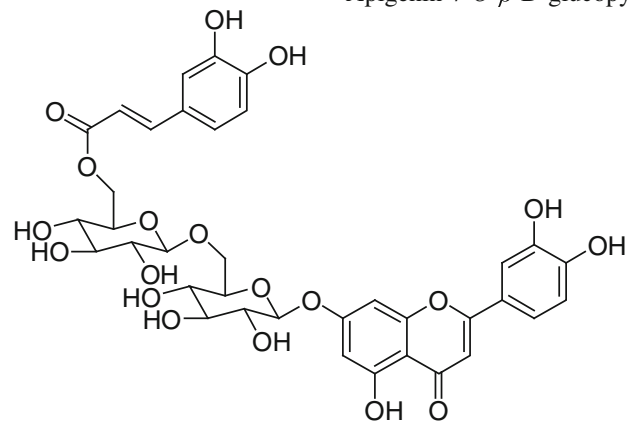

Luteolin-7-O- $\beta$-D-glucopyranosyl $(1 \rightarrow 6)$-[(6"'-O-caffeoyl)]- $\beta$-D-glucopyranoside (93)

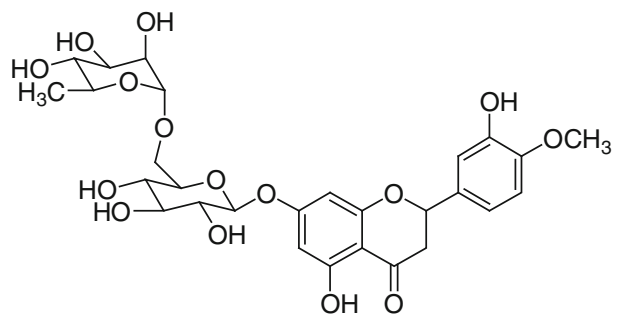

Hesperidin (94)

Fig. 5 Structures of flavones (85-94) from Codonopsis species

molar ratio of 1.13:1.12:1. Its main chain was shown to be $(1 \rightarrow 3)$-linked- $\beta$-GalpNAc, $(1 \rightarrow 3)$-linked- $\alpha$-Rhap and $(1 \rightarrow 2$, 3)- $\beta$-Galp [53]. Furthermore, a pectic polysaccharide with a molecular mass of $1.45 \times 10^{5} \mathrm{Da}$ was at first isolated from C. pilosula, and its structural analysis revealed that this polysaccharide is composed of rhamnose, arabinose, galactose and galacturonic acid in the molar ratio of 0.25:0.12:0.13:2.51. Combined with chemical and spectroscopic analyses, its structure was proposed to be 1,4-linked- $\alpha$-D-GalpA and 1,4-linked- $\alpha$-D-GalpA6Me interspersed with rare 1,2-linked- $\beta$-L-Rhap, 1,2,6-linked- $\alpha$ D-Galp and terminal $\alpha$-L-Arap [54].

\section{Bioactivities}

Although there is information on the uses of many Codonopsis species in traditional medicine, only bioactivity studies on $C$. pilosula and $C$. lanceolata have been reported frequently, which proved their importance as medicinal plants. Bioactivity studies on other Codonopsis species such as $C$. clematidea and $C$. cordifolioidea were scarce. The studies generally referred to the bioactive effects of aqueous, methanol and ethanol extracts, as well as their further purified fractions, flavones, saponins and polysaccharides.

\section{Codonopsis pilosula}

Anti-tumor activity

The polysaccharide from C. pilosula $(10 \mu \mathrm{g} / \mathrm{mL})$ was able to inhibit the activities of human gastric adenocarcinoma cells and hepatoma carcinoma cells [55]. A pectic polysaccharide $(50,100,200$ and $400 \mu \mathrm{g} / \mathrm{mL})$ exhibited marked cytotoxicity to human lung adenocarcinoma A 549 cells, in a dose-dependent manner [54].

Anti-diabetic activity

After mice were orally administered the polysaccharide from $C$. pilosula for a week, Fu et al. [56] found that three different doses of polysaccharide (100, 200 and $300 \mathrm{mg} /$ $\mathrm{kg}$ /day) could effectively decrease fasting blood glucose and insulin in serum, enhance superoxide dismutase (SOD) 
Fig. 6 Structures of organic acids (95-103) from Codonopsis species<smiles>O=C(O)CCC(=O)O</smiles>

Succinic acid (95)<smiles>O=C(/C=C/c1ccc(O)c(O)c1)O[C@@H]1C[C@@](O)(C(=O)O)C[C@H](O)[C@H]1O</smiles><smiles>O=C(/C=C/c1ccc(O)c(O)c1)OC1CC(O)(C(=O)O)C[C@H](O)C1O</smiles><smiles>[R]c1ccc(C(=O)O)cc1[Z17]</smiles>

4-( $\beta$-D-glucopyranosyl)-benzoic acid (98): $\mathrm{R}^{1}=\mathrm{H}, \mathrm{R}^{2}=\mathrm{O}$-Glc Vanillic acid (103): $\mathrm{R}^{1}=\mathrm{OCH}_{3}, \mathrm{R}^{2}=\mathrm{OH}$<smiles>O=C(O)/C=C/c1ccc(O)c(O)c1</smiles>

Caffeic acid (99)

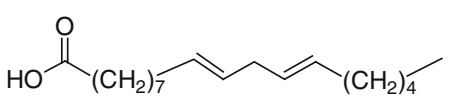<smiles>CCCCCCCCCCC(C)O</smiles><smiles>O=C(O)C1=C[C@@H](O)[C@H](O)[C@H](O)C1</smiles>

Linoleic acid (100)<smiles>C=C1O[C@@]2(O)CC3(C)CCCC(=C)C3CC2=C1C</smiles>
Atractylenolide III (104)<smiles>COC(=O)C1C=CC(CO)C2C(CO)C=CCC12</smiles><smiles>O=Cc1ccc(CO)o1</smiles>
5-Hydroxymethyl-2-furaldehyde (105)<smiles>CCCCCOC1OC(CO)[C@@H](O)[C@H](O)[C@H]1O</smiles>

Hexyl- $\beta$-D-glucopyranoside (110)<smiles>O=c1ccc2ccc3occc3c2o1</smiles>

Angelicin (106)<smiles>CCCCO[C@]1(O)O[C@H](CO)[C@@H](O)[C@H]1O</smiles>

Butyl- $\beta$-D-fructournanoside (111)<smiles>O=c1ccc2cc3ccoc3cc2o1</smiles>
Psoralen (107)<smiles>Cc1cc(O)c2c(c1)C(=O)c1cc(O)cc(O)c1C2=O</smiles>

$$
\widehat{\mathrm{OR}}
$$

Hexyl- $\beta$-gentiobioside (114): $\mathrm{R}=\mathrm{Glc}(1 \rightarrow 6)$-Glc Hexyl- $\beta$-sophoroside (115): $\mathrm{R}=\mathrm{Glc}(1 \rightarrow 2)$-Glc
(E)-2-Hexenyl- $\beta$-sophoroside (116):

$$
\widehat{\mathrm{OR}}
$$

(E)-2-Hexenyl- $\alpha$-L-arabinopyranosyl $(1 \rightarrow 6)-\beta$-D-glucopyranoside (117): $\mathrm{R}=\mathrm{Ara}(1 \rightarrow 6)$-Glc<smiles>[R6]c1cc2c(cc1OC)OC[C@H](c1ccc(O)cc1OC)[C@H]2O</smiles>

Cordifoliflavane A (118): $\mathrm{R}=\mathrm{CH}_{3}$ Cordifoliflavane B (119): $\mathrm{R}=\mathrm{H}$<smiles>COC(C)/C=C/C1C(C)=CC(=O)CC1(C)C</smiles>

3-Oxo- $\alpha$-ionol- $\beta$-D-glucopyranoside (124)<smiles>O=C(CCO)c1cc2occc2cc1O</smiles>

Lanceolune A (120): $\mathrm{R}=\mathrm{H}$ Lanceolune B (121): $\mathrm{R}=\mathrm{CH}$<smiles>C1CCCCC1</smiles><smiles>CO</smiles><smiles>COc1cc2ccoc2cc1/C=C/C(=O)O</smiles>
Lanceolune $\mathrm{C}(\mathbf{1 2 2})$<smiles>COC1=C(C)CCCC1</smiles><smiles>C=CC(=CC)C(CCO)C(CCO)c1ccc(O)c(OC)c1</smiles>

y-3-methoxyphenyl) (125)

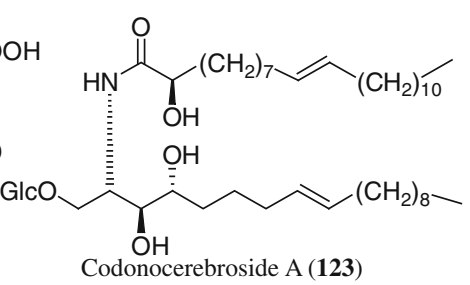<smiles>O=C1OCCC2C1=CO[C@H](Cl)[C@H]2C=S</smiles>

Fig. 7 Structures of other compounds (104-126) from Codonopsis species

activity and reduce the content of malondialdehyde (MDA) in serum. It was therefore considered to possess a significant hypoglycemic effect in diabetic mice by improving insulin resistance. He et al. [57] showed that the aqueous extract of the roots of $C$. pilosula (equal to $4.5 \mathrm{~g}$ raw material $/ \mathrm{kg} /$ day) might retard the progression of diabetes 
by reducing the blood glucose level and preventing the increase of aldose reductase activity in streptozotocininduced diabetic mice after 3 days of oral administration.

Anti-aging activity

Xu et al. [58] found that after mice were orally administrated the polysaccharide from $C$. pilosula for 8 weeks, the polysaccharide (50 and $150 \mathrm{mg} / \mathrm{kg} / \mathrm{day}$ ) was able to increase the thymus index and spleen index as well as the activities of SOD in serum and liver, glutathione peroxidase and nitric oxide synthase particularly in kidney, while decreasing MDA in serum and liver and lipofuscin in brain. Its postponement of senility might be related to raising immunity, eliminating free radicals and anti-lipoperoxidation.

\section{Effects on gastric mucosa}

Liu et al. $[59,60]$ found that the water-soluble fraction from the roots of C. pilosula (equal to $10 \mathrm{~g}$ raw material/ $\mathrm{kg}$ ) had a significant protective effect on gastric mucosal damage caused by alcohol, $0.6 \mathrm{~N} \mathrm{HCl}$ and $0.2 \mathrm{~N} \mathrm{NaOH}$, and suggested that the pharmacological mechanism was related to the synthesis and/or release of prostaglandins in gastric mucosa. To date, Song et al. [61] found that lobetyolin at the oral dose of $1.5 \mathrm{mg} / \mathrm{kg}$ had an effect on decreasing the ulcer index and the level of gastrin and increasing the level of 6-keto-prostaglandin F1 $\alpha$ in rats with gastric ulcer induced by ethanol, and suggested that lobetyolin played a protective role in gastric mucosa injury.

\section{Effects on blood system}

Aqueous extracts of C. pilosula $(500 \mu \mathrm{g} / \mathrm{mL})$ potently inhibited erythrocyte hemolysis [62]. In addition, after ischemia-reperfusion injury rats received $8 \mathrm{mg} / 100 \mathrm{~g}$ body weight of a solution of saponins via intraperitoneal injection, the results showed that the increase in SOD levels was accompanied by decreases in MDA, serum creatinine and blood urea nitrogen levels; $b c l$-2 mRNA and protein levels were raised in transplanted kidneys from treated animals, while bax mRNA and protein levels were reduced. The apoptosis index was significantly decreased in transplanted kidneys from treated animals relative to untreated controls. These results clearly demonstrated protective effects on ischemia-reperfusion injury after kidney transplantation, which might be explained by decreasing lipid peroxidation and inhibition of apoptosis [63].

Effects on immunity

Zhang and Wang [64, 65] found that 6 days of oral administration of the polysaccharide from $C$. pilosula
( $800 \mathrm{mg} / \mathrm{kg} /$ day) had effects on immunosuppressed mice induced by cyclophosphamide, including increasing the thymus and spleen index and the phagocytic activity of peritoneal macrophages and recovering the activity of $\alpha$ naphthyl-acetate esterase in peripheral lymphocytes. In an immunological study in vitro, a water-soluble polysaccharide $(50,100$ and $200 \mu \mathrm{g} / \mathrm{mL})$ could stimulate concanavalin A- or lipopolysaccharide (LPS)-induced lymphocyte proliferation in a dose-dependent manner [52]. In addition, the methanol extract of $C$. pilosula $(1 \mathrm{mg} / \mathrm{mL})$ inhibited inducible nitric oxide synthase and protein oxidation in LPS-stimulated murine RAW 264.7 macrophage cells [66].

Effects on nervous system

Total alkaloids ( $30 \mu \mathrm{g} / \mathrm{mL}$ ) caused a significant enhancement of nerve growth factor-induced neurite outgrowth in PC12 cells as well as an increase in the phosphorylation of mitogenactivated protein kinase [67]. Moreover, Pan et al. [68] orally administered alkaloids from C. pilosula ( $1 \mathrm{mg} / \mathrm{kg} / \mathrm{day})$ to mice for 4 days after they suffered from amnesia by scopolamine, and found that the alkaloids were effective against the decrease in acetylcholine. Other chemicals, saponins from Codonopsis Radix, were reported to have protective effects on the damage to astrocytes induced by hypoxia/hypoglycaemia and reoxygenation, and were able to inhibit the necrosis of astrocytes at three different concentrations (5.2, 52 and $520 \mu \mathrm{g} / \mathrm{mL}$ ) [69]. Additionally, the polysaccharide from $C$. pilosula $(1.1 \mathrm{mmol} / \mathrm{mL})$ also had marked protective effect on neural stem cell injury induced by sodium thiosulphate [70].

\section{Other bioactivities}

The extract of $C$. pilosula (20, 40 and $60 \mu \mathrm{g} / \mathrm{mL}$ ) significantly attenuated angiotensin II (AngII)-induced insulinlike growth factor II receptor (IGFIIR) promoter activity. C. pilosula also reversed $\mathrm{Ca}^{2+}$ influx, mitochondrial outermembrane permeability and apoptosis increased by AngII plus Leu ${ }^{27}$-IGFII which was applied to enhance the AngII effect. Molecular markers in the IGFIIR apoptotic pathway and IGFIIR-Gaq association were down-regulated by $C$. pilosula. However, p-BadSer136 and Bcl-2 were increased. The results suggested that $C$. pilosula could suppress the AngII plus Leu ${ }^{27}$-IGFII-induced IGFII/IGFIIR pathway in myocardial cells [71].

\section{Codonopsis lanceolata}

Antioxidant activity

The water-soluble fraction and the $n$-butanol-soluble fraction of ethanol extract of $C$. lanceolata showed 
significantly anti-oxidative effects at concentrations of 7.3 and $9.3 \mathrm{mg} / \mathrm{mL}$, respectively. The anti-oxidative effects resulted from the free radical scavenging function, while the former had a much stronger effect [72].

\section{Hepatoprotective activity}

After the alcohol-induced hepatic injury mice were orally administered the ethanol extract of $C$. lanceolata ( 1 and $2 \mathrm{~g} / \mathrm{kg} /$ day) for 8 weeks, a protective effect was seen [73]. Zhang et al. suggested that the mechanism might be related to anti-oxidative ability, reduced liver lipid peroxidation, and elimination of free radical and its products [73]. On the other hand, the methanol extract of C. lanceolata $(182 \mathrm{mg} /$ $\mathrm{kg} / \mathrm{day}$ ) appeared to be associated with adenosine- and adiponectin-mediated regulation of hepatic steatosis and Toll-like receptor-mediated modulation of hepatic proinflammatory responses in mice with alcoholic fatty livers after 8 weeks of oral administration [74]. Kim et al. [75] indicated that a week of oral administration of the saponins from C. lanceolata $(50 \mathrm{mg} / \mathrm{kg} / \mathrm{day})$ could decrease the upregulated levels of glutamate-oxalacetate transaminase and glutamate-pyruvate transaminase in serum of mice induced by water-immersed stress conditions, and the hepatic protective effect was marked due to radical scavenging actions.

\section{Anti-inflammatory activity}

Triterpenoids contributed to the anti-inflammatory activity of the ethanol extract of C. lanceolata [76]. Lee et al. [77] have shown that methanol extracts of $C$. lanceolata $(100 \mathrm{mg} / \mathrm{mL})$ clearly blocked the production of TNF- $\alpha$ and nitric oxide, the expression of IL-3 and IL- 6 as well as the phagocytic uptake in LPS-activated RAW264.7 cells, and suggested that the therapeutic activity of $C$. lanceolata on inflammation-mediated symptoms might be mediated by the modulation of macrophage functions. Lancemaside A (15 and $30 \mu \mathrm{M}$ ), a triterpenoid isolated from C. lanceolata, significantly suppressed the inflammatory functions of LPS-treated RAW264.7 cells by suppressing the production of nitric oxide, the expression of the nitric oxide producing enzyme inducible nitric oxide synthase, the upregulation of the costimulatory molecule CD80, and the morphological changes induced by LPS exposure. In addition, lancemaside A diminished the phagocytic activity of RAW264.7 cells and boosted the neutralizing capacity of these cells when treated with the radical generator sodium nitroprusside. Interestingly, lancemaside A strongly blocked the adhesion activity of RAW264.7 cells to plastic culture plates, inhibited the cell-cell adhesion of U937 cells that was triggered by treatment with an anti- $\beta 1$ integrin antibody, and immobilized cell-fibronectin. These findings suggested that the anti-inflammatory mechanism of lancemaside A involves the suppression of the cellular responses of macrophages and monocytes by blocking redox activation and the IKK/NF- $\kappa B$ pathway [78].

Anti-tumor activity

The $n$-butanol soluble fraction of aqueous extract from $C$. lanceolata $(50,100,150$ and $200 \mu \mathrm{g} / \mathrm{mL})$ significantly inhibited human colon cancer HT-29 cell growth in a doseand time-dependent manner by inducing G0/G1 phase arrest and apoptosis [79]. Moreover, the saponins from $C$. lanceolata $(100,150$ and $200 \mu \mathrm{g} / \mathrm{mL})$ could significantly inhibit the growth of HepG2 cells through up-regulating caspases- 8 and caspases- 9 and subsequently activating caspase-3 [80]. Lee et al. [81] indicated that $\beta$-D-xylopyranosyl( $1 \rightarrow 3)$ - $\beta$-D-glucuronopyranosyl echinocystic acid $(40 \mu \mathrm{M})$ was a potent inducer of apoptosis and facilitated its activity via Bid cleavage and translocation to mitochondria, Bax reduction in cytosol, release of cytochrome $c$ and Smac/DIABLO into the cytosol, and subsequently caspase activation.

\section{Effects on blood system}

Byeon et al. [82] found that the $n$-butanol fraction of $C$. lanceolata $(200 \mu \mathrm{g} / \mathrm{mL})$ not only up-regulated mRNA expression of granulocyte-macrophage-colony stimulating factor (GM-CSF) but also increased the proliferation of splenocytes, and suggested that $C$. lanceolata had an effect on recovering the blood loss via activating relevant signaling cascades, such as NF- $\mathrm{KB}$ and AP-1. Choi et al. [83] showed that oral administration of the aqueous extract of C. lanceolata $(100,300$ and $900 \mathrm{mg} / \mathrm{kg} /$ day) for 6 weeks could significantly decrease the weight of adipose pads and the levels of triglycerides, total cholesterol and low-density-lipoprotein cholesterol in rats with high-calorie/highfat-diet-induced obesity. Additionally, 3T3-L1 cells treated with the aqueous extract $(0.5$ and $0.7 \mu \mathrm{g} / \mathrm{mL})$ could also inhibit lipid accumulation and expression of C/EBP $\alpha$ and PPAR $\gamma$. When rats with Qi-deficiency and blood stasis syndrome were orally administered the ethanol extract of C. lanceolata ( 5 and $10 \mathrm{~g} / \mathrm{kg} /$ day) for 14 days, $\mathrm{Xu}$ et al. [84] found that the ethanol extract could decrease whole blood viscosity, plasma viscosity, aggregation indexes of red blood cells and packed red blood cells, and speed up red blood cell electrodeposition time significantly, and concluded that it had effects on invigorating energy, activating blood flow and resolving blood stasis on rats. In addition, after lipid metabolic disorder rats were orally administered the ethanol extract of $C$. lanceolata (equal to $1.5,3.0$ and $6.0 \mathrm{~g}$ raw materials $/ \mathrm{kg} /$ day) for 8 weeks, the results showed that the ethanol extract could decrease the 
ratio of liver weight to body weight, the level of triglyceride and the activity of inducible nitric oxide synthase, and increase nitrogen monoxide, endothelial nitric oxide synthase activity, and the activities of lipases, including total lipase activity in the liver, lipoprotein lipase and hepatic lipase [85]. Han et al. [85] concluded that its mechanism in regulating blood lipids might be by accelerating lipid metabolism and inhibiting the produce of lipid peroxidation.

\section{Effects on nervous system}

After 4 days of oral administration of an ethyl-acetatesoluble fraction of $95 \%$ ethanol extract of C. lanceolata $(50 \mathrm{mg} / \mathrm{kg} / \mathrm{day})$ to gerbils, ischemic neuronal loss was potentially reduced by maintaining $\mathrm{Cu}, \mathrm{Zn}$-superoxide dismutase and brain-derived neurotrophic factor immunoreactivity in the ischemic hippocampal CA1 region [86].

\section{Other bioactivities}

Oleanolic acid isolated from C. lanceolata (20, 40 and $80 \mu \mathrm{g} / \mathrm{mL}$ ) might protect DNA from damage induced by UV radiation and promote DNA damage repair [87].

\section{Codonopsis clematidea}

Chen et al. [88] showed that 15 days of oral administration of polysaccharides from C. clematidea (15 and $30 \mathrm{~g} / \mathrm{kg} /$ day) increased the activities of SOD and reduced the content of MDA in mice brain. Moreover, Han [89] found that 7 days of oral administration of the polysaccharides from C. clematidea $(100 \mathrm{mg} / \mathrm{kg} /$ day $)$ had a significant effect against cancer cachexia in mice and inferred that the possible mechanism might be inhibition of the tumor growth and decrease in the serum content of TNF- $\alpha$ and IL6 . In addition, after the mice were orally administered flavonoids from C. clematidea ( 0.5 and $1.0 \mathrm{mg} / \mathrm{kg} / \mathrm{day})$ for 25 days, Wang et al. [90] found that SOD activities were increased in blood and liver, the content of MDA was decreased in blood and liver, swimming time was prolonged, and the content of liver glycogen and muscle glycogen was increased, and therefore inferred that the flavonoids were related to the antioxidant and anti-fatigue effects of $C$. clematidea.

\section{Codonopsis cordifolioidea}

Qiu et al. [51] showed that the essential oils from fresh roots of $C$. cordifolioidea were effective against Staphylococcus aureus and Salmonella typhimurium with a minimum inhibitory concentration (MIC) of $25 \mu \mathrm{g} / \mathrm{mL}$, and the essential oils from dried roots of $C$. cordifolioidea were effective against Staphylococcus aureus, Salmonella typhimurium, and Shigella flexneri with a MIC of $50 \mu \mathrm{g} /$ $\mathrm{mL}$. Gao et al. [45] showed that 4-hydroxyisoflavanes and cordifoliflavanes A and B isolated from C. cordifolioidea with minimal cytotoxicity against C8166 cells $\left(\mathrm{CC}_{50}>200 \mu \mathrm{g} / \mathrm{mL}\right)$ had weak anti-HIV-1 activities with $\mathrm{EC}_{50}$ values of 5.26 and $2.25 \mu \mathrm{g} / \mathrm{mL}$, respectively, and anti-tobacco mosaic virus activities with inhibition rates of 11.5 and $18.6 \%$, respectively.

\section{Quality control}

Due to the different chemical components, bioactivities and clinical effects of different Codonopsis species, the first step in assuring the quality of traditional medicines is correct identification [91].

To authenticate Codonopsis Radix, morphological and histological studies have been carried out on the roots of seven Codonopsis species [92, 93]. However, their roots could not be identified accurately due to similar morphology. The histological characteristics such as the shape of parenchyma cells of the phloem and xylem, and the percentage value of the diameter of the xylem to that of the root may vary in different growing stages of plants and drying conditions of the roots. On the other hand, Codonopsis Radix consisting of at least two species was commonly found in commodities [94]. Therefore, it is difficult to elucidate the botanical sources of Codonopsis Radix based on morphological inspection and histological characteristics.

DNA-based markers have now become a popular tool for the identification of plants because the genetic composition is unique for each individual and is less affected by growth period, physiological condition, environmental factors, harvest time, storage and processing conditions [95]. In addition, molecular approaches are widely used not only in plant taxonomy but also in identification of crude drugs [94, 96, 97]. Fu et al. [98] reported that the sequence difference allows effective and reliable differentiation of Codonopsis from two related adulterants Campanumoea javania and Platycodon grandiflorus by polymerase chain reactionrestriction fragment length polymorphism (PCR-RFLP). Recently, He et al. [94] have reported that the sequence of the internal transcribed spacer (ITS) of nuclear ribosomal DNA (nrDNA) as a molecular marker could effectively and accurately identify a number of $C$. pilosula, $C$. pilosula var. modesta, $C$. tangshen and $C$. lanceolata specimens and be applied to authentication of Codonopsis Radix.

The diverse chemical components make it possible to discriminate Codonopsis species and further evaluate the 


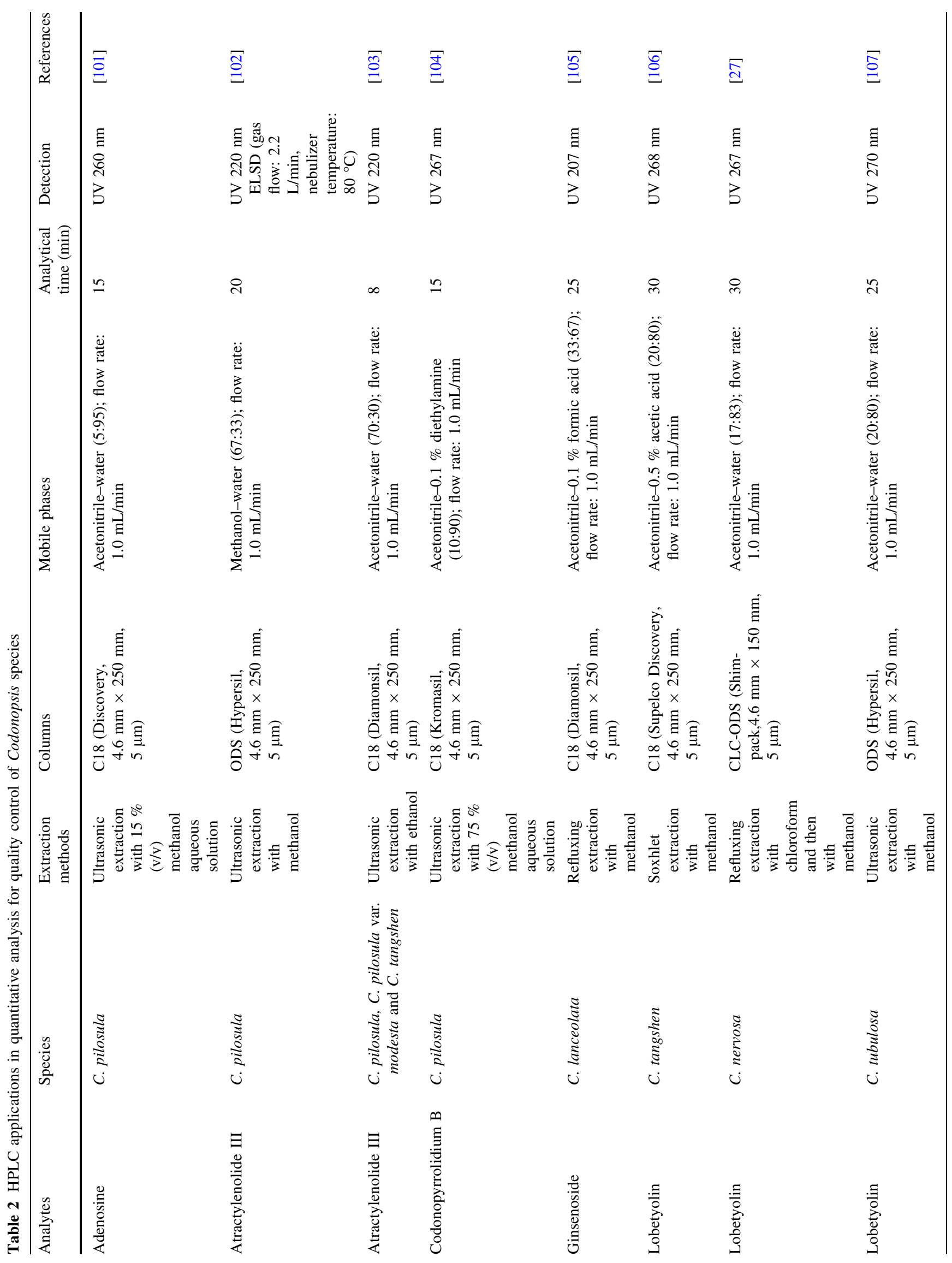




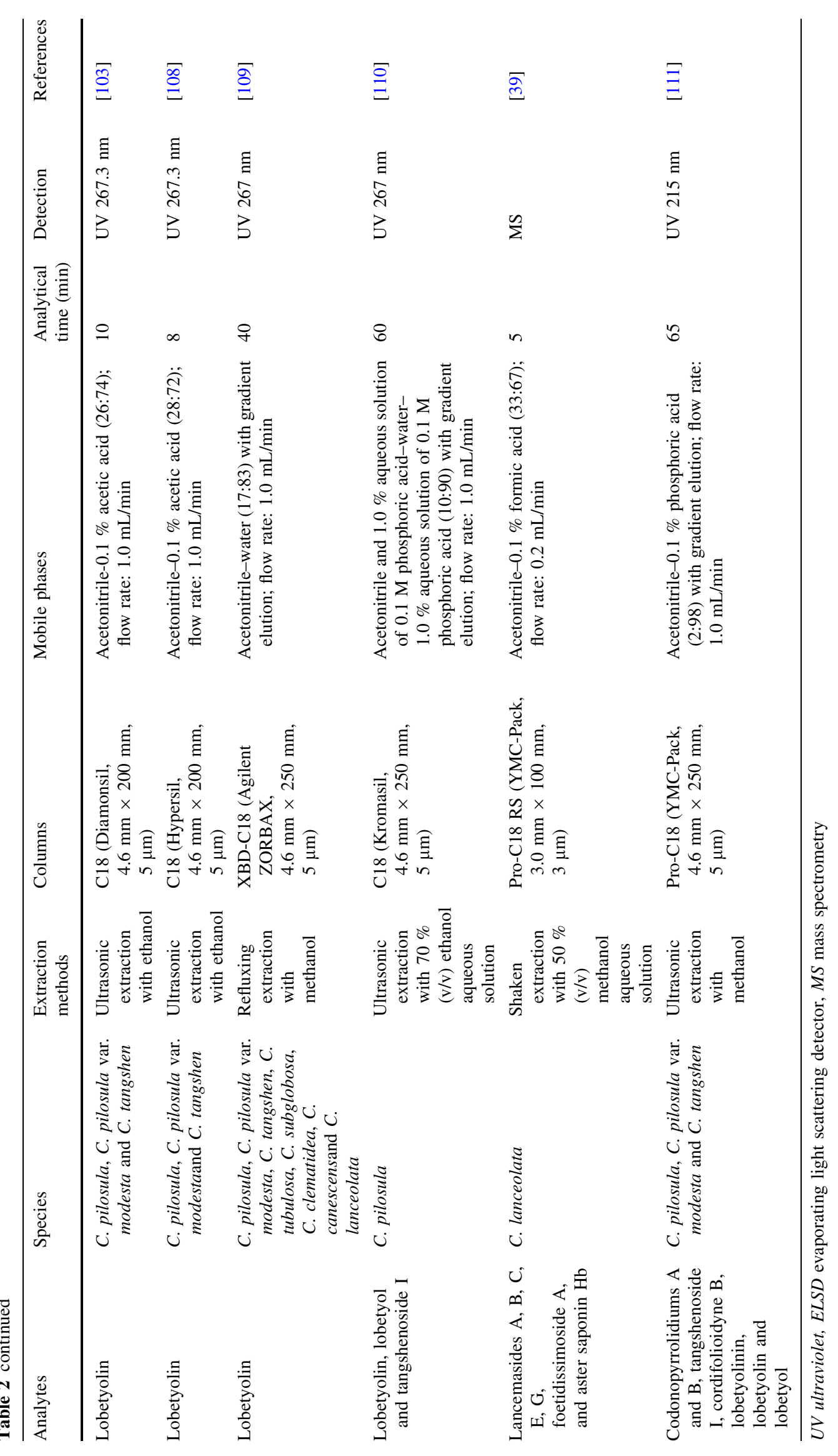


quality of Codonopsis species. Polysaccharides, polyacetylenes, alkaloids and saponins have been proven to contribute to the biological activities of Codonopsis. Therefore, quantitative analysis for the quality control of Codonopsis has mostly focused on these chemotypes of compounds. To date, a series of analytical methods have been employed and reported to quantify the contents of bioactive components in various Codonopsis species. Lu et al. [99] compared the contents of fructose and stigmast7-en-3-ol to discriminate 5 Codonopsis species from each other by thin-layer chromatography (TLC)-ultraviolet spectrum (UV). Tangshenoside I, which was considered as a valuable marker for identification of Codonopsis Radix [23], was detected by TLC-UV [100]. High-performance liquid chromatography (HPLC) is an accurate, sensitive and selective technique which has been widely used in quality control of herbal drugs. Currently, HPLC coupled with UV, evaporating light scattering detector (ELSD) and electrospray ionization (ESI)-mass spectrometry (MS) have become the usual analytical techniques for separation and quantification of markers from complicated Chinese medicinal material extracts. HPLC methods for quantitation of specific chemicals in Codonopsis are summarized in Table 2 [27, 39, 101-111]. It is found that lobetyolin was mostly used as a marker compound for quantitative analysis of Codonopsis Radix. However, Qiao et al. [109] have reported that lobetyolin was widely found not only in the three Codonopsis taxa used as Codonopsis Radix, but also in other species of the same genus and even in species of other genera from the family Campanulaceae, indicating that it was not characteristic for Codonopsis species. Therefore, HPLC methods on the basis of multiple components having potential bioactivities, including polyacetylenes, phenylpropanoids, alkaloids and saponins, were used to compare and assess the quality of Codonopsis species [39, 110, 111]. In addition, chemical fingerprint analysis, which shows not only the bioactive and characteristic components present but also their relative ratios, is also recognized as a reliable means for the identification and qualification of herbal medicines. GC and HPLC-UV fingerprint methods were devised for comparing different Codonopsis species and producing areas of Codonopsis Radix and for evaluating the quality of Codonopsis Radix [109, 112-114]. Moreover, the alkaloids codotubulosines A and $\mathrm{B}$, adenosine and 5-(hydroxymethyl) furfural were analyzed by ${ }^{1} \mathrm{H}$ nuclear magnetic resonance $\left({ }^{1} \mathrm{H}-\mathrm{NMR}\right)$ in 7 Codonopsis species and Campanumoea javanica [15]. The combination of near-infrared (NIR) spectroscopy with chemometrics has been reported to discriminate $C$. pilosula from different geographical origins [115].

Codonopsis Radix is one of the herbs commonly processed by sulfur fumigation, and it is often questioned whether sulfur fumigation could affect the chemical components in the herbs. Ma et al. [116] established a rapid and versatile ultra-high-performance liquid chromatography coupled with ultra-high-resolution quadrupole time-offlight mass spectrometry (UHPLC UHD Q-TOF MS/MS) method that was able to detect 15 sulfur-containing compounds only in sulfur-fumigated samples, and therefore was successfully applied to discriminate sulfur-fumigated Codonopsis Radix among commercial samples.

\section{Conclusion}

The importance of the genus Codonopsis has been highlighted based on their wide uses in traditional medicine. Combining phytochemical investigation of $C$. pilosula, $C$. tangshen and $C$. lanceolata and their widely known bioactive properties, and especially of the related mechanism to explain their ethnomedicinal uses and support development of pharmaceutical products, will undoubtedly be the key for advanced research in the future. Additionally, more studies are still urgently needed on the development of the less popular Codonopsis species. For efficacious and safe use, a series of methods for quality assessment have been developed to ensure the quality of Codonopsis species according to morphological, molecular and chemical characteristics. Establishing a feasible and reliable approach to quality evaluation of various Codonopsis species thus remains difficult, without considering botanical origin, chemical constituents and bioactive activities.

\section{References}

1. Hong DY, Wu ZY, Raven PH (2011) Flora of China, vol 19. Science Press/Missouri Botanical Garden Press, Beijing/St. Louis, pp 513-516

2. Chinese Pharmacopoeia Commission (2010) Pharmacopoeia of the People's Republic of China (2010 edn), vol I. China Medical Science Press, Beijing, p 264

3. Xu GJ, Xu LS (1994) Species systematization and quality evaluation of Chinese traditional drugs, vol 1. Fujian Science and Technology Press, Fuzhou, pp 5-9

4. Nanjing University of Chinese Medicine (2006) Dictionary of Chinese traditional medicine (Zhong Yao Da Ci Dian), 2nd edn. Shanghai Scientific and Technical Publishers, Shanghai, pp 2578-2579

5. Guo WL, Gong L, Ding ZF, Li YD, Li FX, Zhao SP, Liu B (2006) Genomic instability in phenotypically normal regenerants of medicinal plant Codonopsis lanceolata Benth. et Hook. f., as revealed by ISSR and RAPD markers. Plant Cell Rep 25:896-906

6. Wang ZT, Ma GY, Tu PF, Xu GJ, Ng TB (1995) Chemotaxonomic study of Codonopsis (family Campanulaceae) and its related genera. Biochem Syst Ecol 23:809-812

7. Lee KT, Choi J, Jung WT, Nam JH, Jung HJ, Park HJ (2002) Structure of a new echinocystic acid bisdesmoside isolated from 
Codonopsis lanceolata roots and the cytotoxic activity of prosapogenins. J Agric Food Chem 50:4190-4193

8. Ichikawa M, Ohta S, Komoto N, Ushijima M, Kodera Y, Hayama M, Shirota O, Sekita S, Kuroyanagi M (2009) Simultaneous determination of seven saponins in the roots of Codonopsis lanceolata by liquid chromatography-mass spectrometry. J Nat Med 63:52-57

9. Tashkhodzhaev B, Aripova SF, Turgunov KK, Abdilalimov O (2004) Stereochemistry of the alkaloids codonopsine and codonopsinine. Chem Nat Compd 40:618-619

10. Ishida S, Okasaka M, Ramos F, Kashiwada Y, Takaishi Y, Kodzhimatov OK, Ashurmetov O (2008) New alkaloid from the aerial parts of Codonopsis clematidea. J Nat Med 62:236-238

11. Wakana D, Kawahara N, Goda Y (2013) Two new pyrrolidine alkaloids, codonopsinol $\mathrm{C}$ and codonopiloside $\mathrm{A}$, isolated from Codonopsis pilosula. Chem Pharm Bull 61:1315-1317

12. He JY, Zhu S, Goda Y, Cai SQ, Komatsu K (2014) Quality evaluation of medicinally-used Codonopsis species and Codonopsis Radix based on the contents of pyrrolidine alkaloids, phenylpropanoid and polyacetylenes. J Nat Med 68:326-339

13. Tsai TH, Lin LC (2008) Phenolic glycosides and pyrrolidine alkaloids from Codonopsis tangshen. Chem Pharm Bull 56:1546-1550

14. Wakana D, Kawahara N, Goda Y (2011) Three new triterpenyl esters, codonopilates A-C, isolated from Codonopsis pilosula. J Nat Med 65:18-23

15. Li CY, Xu HX, Han QB, Wu TS (2009) Quality assessment of Radix Codonopsis by quantitative nuclear magnetic resonance. J Chromatogr A 1216:2124-2129

16. Chang YK, Ki SY, Han BH (1986) Chemical studies on the alkaloidal constituents of Codonopsis lanceolata. Yakkak Hoeji $30: 1-7$

17. Yoo HH, Baek SH, Park YK, Lee SH, Kim CM, Lee KS, Park MK, Park JH (2002) Quality control of dried roots of Codonopsis lanceolata. Pharmacology 33:85-87

18. He Q, Zhu EY, Wang ZT, Chou GX, Xu LS, Hu ZB (2006) Study on chemical constitutes of Codonopsis pilosula. Chin Pharm J 41:10-12

19. Ushijima M, Komoto N, Sugizono Y, Mizuno I, Sumihiro M, Ichikawa M, Hayama M, Kawahara N, Nakane T, Shirota O, Sekita S, Kuroyanagi M (2008) Triterpene glycosides from the roots of Codonopsis lanceolata. Chem Pharm Bull 56:308-314

20. Liu T, Liang W, Tu G (1988) Perlolyrine: a beta-carboline alkaloid from Codonopsis pilosula. Planta Med 54:472-473

21. Wang ZT, Xu GJ, Hattori M (1988) Constituents of the roots of Codonopsis pilosula. Shoyakugaku Zasshi 42:339

22. Liu XH, Liu Y, Sun XW, Liu YF (2010) Quantitative determination of adenosine in Codonopsis Pilosula by HPLC. Pharm Today 20:13-15

23. Mizutani K, Yuda M, Tanaka O, Saruwatari Y, Jia MR, Ling YK, Pu XF (1988) Tangshenosides I and II from Chuan-Dangshen, the root of Codonopsis tangshen Oliv. Chem Pharm Bull 36:2726-2729

24. Ren J, Lin Z, Yuan Z (2013) Tangshenosides from Codonopsis lanceolata roots. Phytochem Lett 6:567-569

25. Yuda M, Ohtani K, Mizutani K, Kasai R, Tanaka O, Jia MR, Ling YR, Pu XF, Saruwatari YI (1990) Neolignan glycosides from roots of Codonopsis tangshen. Phytochemistry 2:1989-1993

26. Song D, Chou GX, Zhong GY, Wang ZT (2008) Two new phenylpropanoid derivatives from Codonopsis tangshen Oliv. Helv Chim Acta 91:1984-1988

27. Xie GB, Wu C, Fan Q, Zhou XL, Ge-Sang SL (2011) Determination of lobetyolin in Codonopsis nervosa by high performance liquid chromatography. Lishizhen Med Mater Med Res 22:1321-1322
28. Hu QF, Li XS, Huang HT, Mu HX, Tu PF, Li GP (2012) Phenylpropanoids from the roots of Codonopsis cordifolioidea and their biological activities. B Kor Chem Soc 33:278-280

29. Mei RQ, Lu Q, Hu YF, Liu HY, Bao FK, Zhang Y, Cheng YX (2008) Three new polyyne (=polyacetylene) glucosides from the edible roots of Codonopsis cordifolioidea. Helv Chim Acta 91:90-96

30. Liang ZM, Lin J, Yuan Z (2007) Study on the chemical constituents of Codonopsis lanceolata. Chin J Chin Mater Med 32:1363-1364

31. Aga EB, Li HJ, Chen J, Li P (2012) Chemical constituents from the aerial parts of Codonopsis nervosa. Chin J Nat Med $10: 366-369$

32. Jing J, Zhang JF, Li H, Huang S, Shan LH, Zhou XL (2013) Study on chemical constituents of Codonopsis thalictrifolia Wall. var. mollis (Chipp) L. T. Shen. Lishizhen Med Mater Med Res 24:2340-2342

33. Wang WY, Zhao SC, Liu DX (2011) Study on the chemical constituents of Codonopsis lanceolata. J Chin Med Mater 34:553-555

34. Wang JZ, Wang FP (1996) Study on chemical constituents of Codonopsis tangshen. Nat Prod Res Dev 8:8-12

35. Qi HY, Wang R, Liu Y, Shi YP (2011) Studies on the chemical constituents of Codonopsis pilosula. J Chin Med Mater 34:546-548

36. Xu LP, Wang H, Yuan Z (2008) Triterpenoid saponins with antiinflammatory activity from Codonopsis lanceolata. Planta Med $74: 1412-1415$

37. Yuan Z, Liang ZM (2006) A new triterpenoid saponin from Codonopsis lanceolata. Chin Chem Lett 17:1460-1462

38. Zhang L, Ma Y, Yuan Z (2009) A new triterpenoid saponin from the roots of Codonopsis lanceolata. Asian J Tradit Med 4:210-213

39. Ichikawa M, Ohta S, Komoto N, Ushijima M, Kodera Y, Hayama M, Shirota O, Sekita S, Kuroyanagi M (2009) Simultaneous determination of seven saponins in the roots of Codonopsis lanceolata by liquid chromatography-mass spectrometry. J Nat Med 63:52-57

40. Peng C (2010) Study on the chemical constituents of Codonopsis nervosa. Chin Tradit Patent Med 32:1248-1249

41. Zhao B, Ren J, Yuan Z (2013) Isolation of a new cerebroside from Codonopsis lanceolata. Biochem Syst Ecol 46:26-28

42. Trinh TT, Tran VS, Wessjohann L (2003) Chemical constituents of the roots of Codonopsis pilosula. J Chem 41:119-123

43. He Q, Zhu EY, Wang ZT, Xu LS, Hu ZB (2004) Flavones isolated from Codonopsis xundianensis. J Chin Pharm Sci $13: 212-213$

44. Zhu EY, He Q, Wang ZT, Xu LS, Xu GJ (2001) Chemical study on the root of Codonopsis pilosula. J Chin Pharm Univ 32:94-95

45. Gao X, Mu H, Li X, Yang G, Li G, Hu Q (2012) Two new 4-hydroxyisoflavanes from the root of Codonopsis cordifolioi$d e a$ and their anti-virus activities. J Chin Chem Soc 59:540-543

46. Hu QF, Li XS, Huang HT, Mu HX, Tu PF, Li GP (2012) New benzofuranylpropanoids from the roots of Codonopsis lanceolata. Helv Chim Acta 95:349-352

47. Jiang PF, Yan YH, Zhao ZJ, Jiang WQ (1992) Analysis of the nutritive constituents in the aerial parts of Codonopsis pilosula. Nat Prod Res Dev 2:31-35

48. Li C, Zhang H, Peng L, Xiang JH, Dong WY, Yang MZ, Liang XY, Kong FH, Zhang ZJ (1993) Study of volatile chemical components in Codonopsis pilosula. J Yunnan Univ 15:86-90

49. Chen M, Li XJ, Jiang L, Yu JX, Liu FM (2000) Study on the essential oils Codonopsis clematidea. Chin Tradit Herb Drugs 31:254-255

50. Liu X, Bai Y, Da-wa ZM, Bai BR, Gu YC (2008) Analysis of the essential oil composition from traditional Tibetan medicine 
of Codonopsis thalictrifolia Wall. by GC-MS. J Instrum Anal 27:86-87

51. Qiu B, Lv Q, Bao FK, Zhang CJ, Cheng YX (2010) GC-MS analysis and antimicrobial activity of essential oils from the fresh and dried roots of Codonopsis cordifolioidea. Nat Prod Res Dev 22:445-449

52. Sun YX, Liu JC (2008) Structural characterization of a watersoluble polysaccharide from the roots of Codonopsis pilosula and its immunity activity. Int J Biol Macromol 43:279-282

53. Zhang YJ, Zhang LX, Yang JF, Liang ZY (2010) Structure analysis of water-soluble polysaccharide CPPS3 isolated from Codonopsis pilosula. Fitoterapia 81:157-161

54. Yang C, Gou Y, Chen J, An J, Chen W, Hu F (2013) Structural characterization and antitumor activity of a pectic polysaccharide from Codonopsis pilosula. Carbohydr Polym 98:886-895

55. Yang FR, Li ZM, Gao JP (2011) Separation and structural characterization and anti-tumor effect in vitro of polysaccharides from Radix Codonopsis. Lishizhen Med Mater Med Res 22:2876-2878

56. Fu PP, Hong T, Yang Z (2008) Effect of polysaccharides from Radix Codonopsis on insulin resistance in diabetic mice. Lishizhen Med Mater Med Res 19:2414-2416

57. He K, Li XG, Chen X, Ye XL, Huang J, Jin YN, Li PP, Deng YF, Jin Q, Shi Q, Shu HJ (2011) Evaluation of antidiabetic potential of selected traditional Chinese medicines in STZinduced diabetic mice. J Ethnopharmacol 137:1135-1142

58. Xu AX, Zhang ZM, Ge B, Pu JF (2006) Study effect and its mechanism on resisting senility of Codonopsis pilosula Nannf. Chin J Mod Appl Pharm 23:729-731

59. Liu L, Wang JH, Hou N (1989) Studies on the pharmacological effects and mechanisms of Codonopsis pilosula (CP) and its efficacious chemical ingredients on preventing the gastric mucosal damage of rats. I The effects of the decoction of CP. Pharmacol Clinics Chin Mater Med 5:11-14

60. Liu L, Wang JH, Hu Y, Hou N, Chen JW (1990) Studies on the pharmacological effects and mechanisms of Codonopsis pilosula $(\mathrm{CP})$ and its efficacious chemical ingredients on preventing the gastric mucosal damage of rats. III The effects of factions extracted from the section VII of CP. Pharmacol Clinics Chin Mater Med 6:20-23

61. Song D, Wang ZT, Li LY, Zhong GY (2008) Protective effect of Lobetyolin on gastric mucosa of experimental gastric ulcer in rats. J Emerg Tradit Chin Med 17:963-964

62. Ng TB, Liu F, Wang HX (2004) The antioxidant effects of aqueous and organic extracts of Panax quinquefolium, Panax notoginseng, Codonopsis pilosula, Pseudostellaria heterophylla and Glehnia littoralis. J Ethnopharmacol 93:285-288

63. He B, Zhang YT, Yuan XG, Sun JS, Wei GH, Lin T (2011) Protective effects of Radix Codonopsis on ischemia-reperfusion injury in rats after kidney transplantation. Pediatr Surg Int 27:1203-1212

64. Zhang RX, Wang FL (1992) Regulatory effect of CPPS on cellmediated immunity in mice. J Lanzhou Med Coll 18:161-165

65. Zhang RX, Wang FL (1993) Effect of CPPS on humoral immunity and IL-2 production. J Lanzhou Med Coll 19:14-17

66. Yoo CS, Kim SJ (2013) Methanol extract of Codonopsis pilosula inhibits inducible nitric oxide synthase and protein oxidation in lipopolysaccharide-stimulated raw cells. Tropical J Pharm Res 12:705-710

67. Liu JH, Bao YM, Song JJ, An LJ (2003) Codonopsis pilosula (Franch.) Nannf. total alkaloids potentiate neurite outgrowth induced by nerve growth factor in PC12 cells. Acta Pharmacol Sin 24:913-917

68. Pan SY, Zhang YF, Liu Y, Xu QP, Jiang MY, Guo YJ (1992) Effects of alkaloids of Dangshen (Codonopsis pilosula) on amnesia induced by scopolamine and the acetylcholine and choline acetyltransferase of brain in mice. Pharmacol Clinics Chin Mater Med 8:22-24

69. Yan YF, Zhang Z, Wei Y, Niu FL, Cui W, Wang SR, Sun SL, Zhu LQ (2006) Preventive effect of total saponin of Radix Codonopsis on Ast lesion induced by hypoxia and hypoglycemia reoxygenation in rats. $\mathbf{J}$ Beijing Univ Tradit Chin Med 29:826-829

70. Wu BF, Yang J, Xie H, Yang XS (2008) Protective effect of polysaccharides from Codonopsis Pilosula on neural stem cell injury induced by $\mathrm{Na}_{2} \mathrm{~S}_{2} \mathrm{O}_{3}$. Lishizhen Med Mater Med Res 19:280-281

71. Tsai KH, Lee NH, Chen GY, Hu WS, Tsai CY, Chang MH, Jong GP, Kuo CH, Tzang BS, Tsai FJ, Tsai CH, Huang CY (2013) Dung-shen (Codonopsis pilosula) attenuated the cardiacimpaired insulin-like growth factor II receptor pathway on myocardial cells. Food Chem 138:1856-1867

72. Li XS, Kuto S, Kanazawa I (2007) Biochemical effects of Codonopsis lanceolata extract. W China J Pharm Sci 22:306-307

73. Zhang L, Han CJ, Li LJ, Tao L, Piao JM (2007) Protective effect of Codonopsis lanceolata extract on alcoholic hepatic injury. Zhongguo Zuzhi Gongcheng Yanjiu yu Linchuang Kangfu 11:5742-5744

74. Cha A, Choi Y, Jin Y, Sung MK, Koo YC, Lee KW, Park T (2012) Antilipogenic and anti-inflammatory activities of Codonopsis lanceolata in mice hepatic tissues after chronic ethanol feeding. J Biomed Biotechnol 2012:141395

75. Kim MH, Lee J, Yoo DS, Lee YG, Byeon SE, Hong EK, Cho JY (2009) Protective effect of stress-induced liver damage by saponin fraction from Codonopsis lanceolata. Arch Pharm Res 32:1441-1446

76. Xu LP, Wang H, Yuan Z (2008) Triterpenoid saponins with antiinflammatory activity from Codonopsis lanceolata. Planta Med 74:1412-1415

77. Lee YG, Kim JY, Lee JY, Byeon SE, Hong EK, Lee J, Rhee MH, Park HJ, Cho JY (2007) Regulatory effects of Codonopsis lanceolata on macrophage-mediated immune responses. J Ethnopharmacol 112:180-188

78. Kim E, Yang WS, Kim JH, Park JG, Kim HG, Ko J, Hong YD, Rho HS, Shin SS, Sung GH, Cho JY (2014) Lancemaside A from Codonopsis lanceolata modulates the inflammatory responses mediated by monocytes and macrophages. Mediat Inflamm 2014:405158

79. Wang L, Xu ML, Hu JH, Rasmussen SK, Wang MH (2011) Codonopsis lanceolata extract induces G0/G1 arrest and apoptosis in human colon tumor HT-29 cells-involvement of ROS generation and polyamine depletion. Food Chem Toxicol 49:149-154

80. Yu X, Li L, Han CJ, Zhang QG (2011) Effects of Codonopsis lanceolata total sanponin on apoptosis in HepG-2 cells. J Jilin Univ (Med Edit) 37:1090-1093

81. Lee KW, Jung HJ, Park HJ, Kim DG, Lee JY, Lee KT (2005) $\beta$ D-Xylopyranosyl-(1-3)- $\beta$-D-glucuronopyranosyl echinocystic acid isolated from the roots of Codonopsis lanceolata induces caspase-dependent apoptosis in human acute promyelocytic leukemia HL-60 cells. Biol Pharm Bull 28:854-859

82. Byeon SE, Lee YG, Cho JY (2009) Regulatory effects of Codonopsis lanceolata on gene expression of GM-CSF in macrophage-like cells. J Ethnopharmacol 123:185-189

83. Choi HK, Won EK, Jang YP, Choung SY (2013) Antiobesity effect of Codonopsis lanceolata in high-calorie/high-fat-dietinduced obese rats. Evid-based Complement Altern Med 2013:210-297

84. Xu Q, Chen XJ, Liu BM, Yang P, Bai XQ, Deng LD, Chen XF (2008) Studies on invigorating energy, activating blood flow, resolving blood stasis and anti-aging actions of alcohol extracts of Codonopsis lanceolata. Guangxi Med J 30:1834-1837 
85. Han CJ, Wang DM, Yu X, Li LJ, Piao YQ (2005) Preventive effect of Codonopsis lanceolata on lipid metabolic disorder in rats and its mechanism. J Jilin Univ (Med Edi) 31:564-566

86. Yoo KY, Lee CH, Li H, Park JH, Choi JH, Hwang IK, Kang IJ, Won MH (2011) Ethyl acetate extracts of raw and steamed Codonopsis lanceolata protects against ischemic damage potentially by maintaining SOD1 and BDNF levels. Int J Neurosci 121:503-509

87. Zhao JH, Han CJ, Yu X, Piao HS (2012) Protactive effects of oleanolic acid isolated from the roots of Codonopsis lanceolata for DNA damage induced by ultraviolet ray. J Med Sci Yanbian Univ 35:22-24

88. Chen M, Xiong YJ, Li XJ, Shen SK, Chen JD (2000) Effect of clematis asiabell (Codonopsis clematidea) and pilose asiabell (Codonopsis pilosula) on SOD and MDA of mouse brain. Chin Tradit Herb Drugs 31:280-281

89. Han L (2010) Experimental study of polysaccharides of Sinkiang lanceolata on mice with cancer cachexin. J N Pharm $7: 17-18$

90. Wang JH, Yuan H, Li XH (2012) Antioxidant and antifatigue activities of flavonoid from Codonopsis clematidea (Schrenk). Nat Prod Res Dev 24:1035-1039

91. World Health Organization (2000) WHO general guidelines for methodologies on research and evaluation of traditional medicine. http://whqlibdoc.who.int/hq/2000/WHO_EDM_TRM_ 2000.1.pdf Accessed 25 July 2014

92. Namba T, Komatsu K, Iwai M, Xu GJ (1992) Pharmacognostical studies on the Codonopsis plants (1). On the anatomical characteristics of the root of Sect. Codonopsis plants. Shoyakugaku Zasshi 46:156-164

93. Namba T, Komatsu K, Iwai M (1992) Pharmacognostical studieson the Codonopsis plants (2). On the botanical origin of the Chinese crude drug "Dangshen" and its related crude drugs derived from Sect. Codonopsis plants. Shoyakugaku Zasshi 46:165-173

94. He JY, Zhu S, Komatsu K, Goda Y, Cai SQ (2014) Genetic polymorphism of medicinally-used Codonopsis species in an internal transcribed spacer sequence of nuclear ribosomal DNA and its application to authenticate Codonopsis Radix. J Nat Med 68:112-124

95. Balasubramani SP, Goraya GS, Venkatasubramanian P (2011) Development of ITS sequence-based markers to distinguish Berberis aristata DC. from B. lycium Royle and B. asiatica Roxb. 3. Biotechnology 1:11-19

96. Sukrong S, Zhu S, Ruangrungsi N, Phadungcharoen T, Palanuvej C, Komatsu K (2007) Molecular analysis of the genus Mitragyna existing in Thailand based on rDNA ITS sequences and its application to identify a narcotic species: Mitragyna speciosa. Biol Pharm Bull 30:1284-1288

97. Balasubramani SP, Murugan R, Ravikumar K, Venkatasubramanian P (2010) Development of ITS sequence based molecular marker to distinguish, Tribulus terrestris L. (Zygophyllaceae) from its adulterants. Fitoterapia 81:503-508

98. Fu RZ, Wang J, Zhang YB, Wang ZT, But PP, Li N, Shaw PC (1999) Differentiation of medicinal Codonopsis species from adulterants by polymerase chain reaction-restriction fragment length polymorphism. Planta Med 65:648-650

99. Lu YR, Yang XY, Sha DZ (1989) Assay of stigmast-7-en-3-ol and fructose in five Codonopsis species. Chin J Chin Mater Med $14: 36-38$
100. Han GR, Wang CP, Su XG, He XF, Wang YD, Mizutani K, Tanaka O (1990) Determination of tangshenoside I in Codonopsis pilosula Nannf. by TLC-UV spectrophotometric method. Chin J Chin Mater Med 15:553-557

101. Liu XH, Liu Y, Sun XW, Liu YF (2010) Quantitative determination of adenosine in Codonopsis Pilosula by HPLC. Pharm Today 20:13-15

102. Li ZH, Hu Y, Liu Q (2005) Comparison of the contents of a tractylenoide III in Dangshen that two detectors determined. J Gansu Coll Tradit Chin Med 22:45-47

103. Pang WR, Shuang SM, Liu YQ (2008) RP-HPLC determination of the contents and correlation of atractylenolide and lobetyolin. World J Integr Tradit West Med 3:89-91

104. Cao YK, Lin ZZ, Chen DJ, Ning Y, Zhu CC (2012) Determination of codotubulosine A in Radix Codonopsis by HPLC. Tradit Chin Drug Res Clin Pharmacol 23:678-680

105. Chen HC, Yuan Z (2010) Study on quality standard of Codonopsis lanceolata. China Pharm 21:2172-2174

106. Song D, Cheng XM, Li LY, Zhong GY, Wang ZT (2008) Determination of lobetyolin in root of Codonopsis tangshen from various cultivation areas by high performance liquid chromatography. Chin J Chin Mater Med 33:2133-2135

107. Sun QW, He SZ, Huang M (2007) Determination of lobetyolin in Radix Codonopsis Tubulosae of Guizhou. Lishizhen Med Mater Med Res 18:1931-1932

108. Zhao XH, Liu YQ, Wang RS, Guo JL, Zhao P (2007) RP-HPLC analysis of lobetyolin in different parts of Codonopsis pilosula. Chin Tradit Patent Med 29:1046-1047

109. Qiao CF, He ZD, Han QB, Xu HX, Jiang RW, Li SL, Zhang YB, But PPH, Shaw PC (2007) The use of lobetyolin and HPLC-UV fingerprints for quality assessment of Radix Codonopsis. J Food Drug Anal 15:258-264

110. Kim EY, Kim JA, Jeon HJ, Kim S, Kim YH, Kim HY, Whang WK (2014) Chemical fingerprinting of Codonopsis pilosula and simultaneous analysis of its major components by HPLC-UV. Arch Pharm Res. doi:10.1007/s12272-014-0335-3

111. He JY, Zhu S, Komatsu K (2014) HPLC/UV analysis of polyacetylenes, phenylpropanoid and pyrrolidine alkaloids in medicinally used Codonopsis species. Phytochem Anal 25:213-219

112. Feng SL, Hu FD, Liu X, Zhao JX (2005) Chromatography fingerprint of Baitiao Radix Codonopsis in Gansu Province by HPLC. Chin Tradit Pantent Med 27:745-748

113. Song D, Cheng XM, Li LY, Zhong GY, Wang ZT (2008) Study on HPLC fingerprint of Codonopsis tangshen Oliv. Chin Pharm J 43:1136-1139

114. Chen FF, Qi HY, Shi YP (2013) Fingerprint analysis of Codonopsis Radix by HPLC coupled with chemometrics analysis. Chin Herb Med 5:307-312

115. Li BX, Wei YH, Duan HG, Xi LL, Wu XN (2012) Discrimination of the geographical origin of Codonopsis pilosula using near infrared diffuse reflection spectroscopy coupled with random forests and k-nearest neighbor methods. Vib Spectrosc 62:17-22

116. Ma XQ, Leung AK, Chan CL, Su T, Li WD, Li SM, Fong DW, $\mathrm{Yu}$ ZL (2014) UHPLC UHD Q-TOF MS/MS analysis of the impact of sulfur fumigation on the chemical profile of Codonopsis Radix (Dangshen). Analyst 139:505-516 